\title{
Selective adaptation of English consonants using real speech
}

\author{
RONALD A. COLE \\ Carnegie-Mellon University, Pittsburgh, Pennsylvania 15213 \\ WILLIAM E. COOPER \\ Massachusetts Institute of Technology, Cambridge, Massachusetts 02139 \\ and \\ JOEL SINGER and FRAN ALLARD \\ University of Waterloo, Waterloo, Ontario, Canada
}

\begin{abstract}
Most English consonant-vowel (CV) syllables have other CV syllables embedded within them. For example, splicing sufficient energy from the onset of $\left[\mathrm{k}_{\mathrm{a}}\right.$ ] yields [ $\mathrm{p}_{\mathrm{a}}$ ], splicing into [ma] or [va] yields [ba], and splicing into [ja] yields [da]. We spliced successively longer segments from naturally spoken CV syllables to produce sequences of CV syllables which varied in discrete acoustic steps from [ $\mathrm{h}_{\mathrm{a}}$ ] to [ $\mathrm{ph}_{\mathrm{a}}$ ], from [ja] to [da], from [ma] to [ba], from [fa] to [ba], and from [va] to [ba]. Random presentation of syllables in each series resulted in identification functions with typically sharp phoneme boundaries. For example, in the seven-syllable [ja]-[da] series (where [ja] was the original or first syllable), there were $98 \%$ [ja] responses to Syllable 3, but only $12 \%$ [ja] responses $(88 \%$ [da] responses) to Syllable 5. Following the identification test, subjects listened to 180 repetitions of either the first or last syllable in the test series, and were again required to identify randomly presented syllables from the test series. A shift in the phoneme boundary toward the repeated (adapting) syllable was observed for 11 of the 12 repeated syllables. Repeated presentation of [ja], for example, resulted in fewer [ja] responses to syllables in the [ja]-[da] series, compared to performance on the previous identification test. Likewise, repeated listening to [da] resulted in a decrease in [da] responses. Adaptation was selective in that syllables near the phoneme boundary were most affected by the adapting syllable. A shift in the phoneme boundary was also observed for two different continua when the adapting stimulus contained an acoustic feature identical to syllables in the test series. Thus, selective adaptation was found along a [ma]-[ba] continuum following repeated presentation of [na] and following repeated presentation of nasal resonance removed from its syllable context. A second major result, observed in five different experiments, was an asymmetrical adaptation effect. A greater shift in the phoneme boundary was observed following repeated presentation of the first syllable in each series (e.g., [ja]) than for the final embedded syllable (e.g., [da]). The results were discussed in terms of two different models of selective adaptation.
\end{abstract}

Eimas and Corbit (1973) first reported evidence for selective adaptation of speech sounds. In their experiment, subjects were randomly presented with stimuli from a series which varied in discrete acoustic steps from a voiced to voiceless stop consonant (e.g., [b] to [p] or [d] to [t]). Stimuli varied in 5-msec steps in terms of their voice onset time (VOT). VOT is defined as the interval between the onset of the stop burst and the onset of voicing. Stimuli ranged from 0 -msec VOT (a distinct [b]) to 60-msec VOT (a distinct [p]). A stimulus with a VOT of $30 \mathrm{msec}$ was heard equally often as [b] and [p], and this stimulus defined the phoneme boundary.

This research was supported by a grant from the National Research Council of Canada to the first author, and by Research Grant MH-07722, from the National Institute of Mental Health, and National Institute of Mental Health Grant MH-26612 to the second author. We thank Peter Boos and Irv Freilich for the day-to-day running of the experiments. Reprint requests should be sent to Carnegie-Mellon University, Department of Psychology, Prttsburgh. Pennsylvania 15213.
Eimas and Corbit found that repeated presentation of a stimulus from one end of the continuum resulted in a shift of the phoneme boundary toward the adapting stimulus. Repeated presentation of [ba], for example, resulted in fewer [ba] responses for stimuli near the boundary. Their results cannot be easily explained in terms of response bias, since adaptation along a bilabial voiced-voiceless continuum (i.e., [b]-[p]) was produced using an apical stop (i.e., [d] or [t]) as the adapting stimulus. Thus, adaptation with the voiced stop [d] shifted the boundary toward the voiced stop $[b]$ in $a[b]-[p]$ series. This result suggests that a phonetic feature was adapted, and not a particular consonant.

Selective adaptation has also been demonstrated along the place of articulation dimension by Cooper $(1947 a)$. Cooper varied the starting frequency of the second and third formant transitions to produce a series of synthetic $C V$ syllables which varied in discrete steps from $[\mathrm{b}]$ to $[\mathrm{d}]$ to $[\mathrm{g}]$. Adaptation with 
[b] shifted the [b]-[d] boundary toward [b], but did not significantly affect the [d]-[g] boundary. Similarly, adaptation with $[\mathrm{g}]$ shifted the [d]-[g] boundary toward $[\mathrm{g}]$, but did not affect the [b]-[d] boundary. Finally, adaptation with [d] shifted both boundaries toward [d]. From these results, Cooper argued for the existence of a bilabial, apical, and velar subset of detectors for the place of articulation feature.

A number of recent experiments have examined the selective adaptation phenomenon (Ades, 1974a, b; Cooper, 1947a, b; Cooper \& Blumstein, 1974). The research strategy to date has been to examine the effect of different adapting stimuli on a particular test series. For example, Cooper and Blumstein (1974) presented subjects with five real speech adapting stimuli-[bæe, [mae], [vae], [wa], [pha]-and tested for adaptation along a [bae]-[da]-[gae] series. Syllables in this series differed only in the starting frequency of the vowel transitions. Cooper and Blumstein found a large and nearly identical decrease in [b] responses on the [bae]-[dae]-[gae] series following repeated presentation of [bac], [mae], and [vae]. A much smaller, nonsignificant, shift in the phoneme boundary was observed for [ $\mathrm{ph}_{\mathfrak{2}}$ ], and no consistent adaptation effect was observed for [wae] as the adapting syllable. These results support the existence of a finely tuned transition detector, since [bae], [mae], and [vae] have nearly identical vowel transitions, as shown in Figure 1, while [ $\mathrm{ph}_{\mathrm{h}}$ ] has weak vowel transitions and [wae] has more gradual transitions than [bae], [mae], or [vae].

It is clear that the selective adaptation procedure, first applied to speech perception by Eimas and

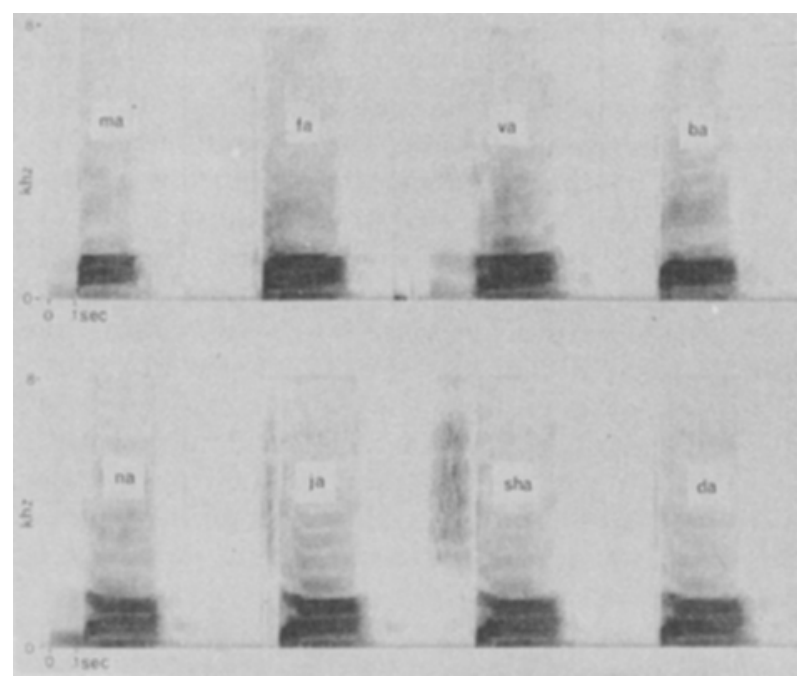

Figure 1. Speech spectrograms of consonant-vowel syllables. Syllables in the top panel have a rising second formant transition since the consonant is articulated toward the front of the mouth. Syllables in the bottom panel, articulated toward the center of the mouth, have a falling second formant transition.
Corbit (1973), provides an exciting paradigm for investigating properties of hypothetical feature detectors. However, because this procedure requires a series of stimuli which vary in precise acoustic steps, all of the experiments to date have been restricted to series of synthetic stop consonants which differ along the voiced-voiceless dimension or the place of articulation dimension. Although research in speech perception has an honorable history of experimentation with synthetic speech, one might hope that experiments in selective adaptation could be performed using real speech stimuli for both the test series and the adapting stimuli.

The present paper describes a series of experiments that used the selective adaptation procedure with real speech stimuli. In all of the experiments described below, the adapting syllable and the series of test syllables were constructed from naturally spoken $\mathrm{CV}$ syllables.

The construction of a variety of different test series was made possible by the fact that most English consonants in prevocalic position have other consonants "embedded" within them (Cole \& Scott, 1974a; Gerstman, 1957; Grimm, 1966; Huntington \& Miller, 1972; Scott, 1971; Carlson, Granstrom, \& Pauli, Note 1). In general, consonants articulated in the front of the mouth contain an embedded [b], while consonants articulated toward the center of the mouth have an embedded [d]. The reason for this can be seen in Figure 1. The top panel in Figure 1 shows four CV syllables articulated at the front of the mouth-[ma], [fa], [va], and [ba]. Each of these syllables has a distinctive initial portion of energy which serves to distinguish the consonant phoneme from the others in the same row. Thus $[\mathrm{m}]$ is accompanied by resonance at the lowest frequencies and a lack of energy in the higher frequencies, [f] is accompanied by low-intensity frication, [v] is accompanied by low-intensity frication plus voicing, and [b] is characterized by an abrupt stop burst and rising transitions to the first and second vowel formants. An examination of the four syllables in the top panel of Figure 1 reveals that all of these syllables have rising transitions to the steady-state vowel. Since [m], [f], $[v]$, and $[b]$ share virtually the same place of articulation, the first and second formant transitions for these syllables are virtually identical.

It is known from experiments with synthetic speech that a syllable composed solely of a rising first (lower) formant transition and a second formant transition pointing to about $700 \mathrm{~Hz}$, followed by steady-state formants, is heard as a CV syllable beginning with the stop consonant [b]. Since precisely this configuration of transitions plus steady-state vowel is embedded within each of the syllables in the top panel of Figure 1, each of these syllables contain an embedded [b]. In fact, when the initial energy is removed from these syllables prior to the onset of the vowel 
transitions, a clear [ba] is heard (Scott, 1971), and this [ba] is operationally defined as an embedded syllable.

Similarly, the four syllables displayed in the bottom panel of Figure 1 each consist of an initial portion of energy typical of the consonant plus an embedded transition-vowel unit. Since [na], [ja], [ša], and [da] are articulated toward the center of the mouth, the second formant transition in each syllable has a starting frequency pointing to about $1,800 \mathrm{~Hz}$, and this is known to cue the stop consonant [d]. Thus, each of the syllables in the bottom panel of Figure 1 has the syllable [da] embedded within it. Removal of the initial resonant energy from [na], or the fricative noise from before [ja] or [sa], or the stop burst from [da] yields, in each case, a perfectly clear [da] cued by the transition-vowel unit.

Because of the presence of embedded sounds, it is possible to construct a series of syllables starting with almost any English CV syllable simply by splicing successive segments off the onset of the syllable until a new embedded $\mathrm{CV}$ syllable is heard. ${ }^{1}$ Thus, we may construct continua for CV syllables in the top panel of Figure 1 from [ma] to [ba], from [fa] to [ba], or from [va] to [ba] by splicing increasingly longer segments off the onset of the original syllable. Similarly, [na]-[da] or [ja]-[da] continua can be constructed by splicing into [na] or [ja]. The present experiments examine selective adaptation for continua constructed from naturally spoken stop, affricate, nasal, and fricative consonants.

\section{EXPERIMENT I}

A number of investigators have observed that the voiceless stop $[\mathrm{p}]$ is embedded in both $[\mathrm{k}]$ and $[\mathrm{t}]$ (Scott, 1971; Carlson, Granstrom, \& Pauli, Note 1). We have recorded [k] before different vowels on several occasions and, after cross-recording the original syllable a number of times, removed successively longer segments from the onset of the $[\mathrm{k}]$. A small percentage of the time, $[\mathrm{k}]$ changes to $[\mathrm{t}]$ and then to [p]. In most cases, a clear change from [k] to [p] is heard without an intervening [t]. We chose a series which changed from [ $\left.k h_{a}\right]$ directly to $\left(\mathrm{p}^{h_{a}}\right]$ (velar to labial), since previous research on the place dimension with synthetic speech had always employed series of voiced stops which progressed from labial to apical or apical to velar but never directly from labial to velar. We were particularly interested in whether selective adaptation would occur in a velar to labial series, since Cooper (1974a) and Cooper and Blumstein (1974), working with synthetic speech, showed that selective adaptation occurred only within a labial-apical ([b]-[d]) or apical-velar ([d]-[g]) series.

\section{Method}

Subjects. Sixteen students from an introductory psychology course served as volunteer subjects. Each student participated in two 45-min sessions 2 days apart. The subjects were tested in groups of three, four, or five in a sound-attenuated room with the experimenter. Each subject was paid $\$ 2$ for his participation. The data for six subjects were not included in the main analyses because of inconsistent responses to stimuli during the identification test. The results for these subjects are discussed separately below.

Stimuli. A naturally spoken $\left[k^{\mathrm{h}} \mathrm{a}\right.$ ] was recorded by a male speaker, and the duration of the consonant was measured from burst onset to the first glottal pulse. The duration of the consonant (burst plus aspiration) was $125 \mathrm{msec}$, with a vowel duration of approximately $300 \mathrm{msec}$. The original $\left[\mathrm{k}_{\mathrm{a}}\right.$ ] was then cross-recorded 11 times and a series was made from $\left[\mathrm{k}_{\mathrm{a}}\right.$ ] to $\left[\mathrm{ph}_{\mathrm{a}}\right]$ by splicing successively longer segments from the onset of each syllable (starting with the second syllable) in 9-msec steps. Thus, the 1st syllable in the series had a consonant duration of $125 \mathrm{msec}$, the 2nd syllable a consonant duration of $116 \mathrm{msec}$, and the 11th syllable had a consonant duration of $35 \mathrm{msec}$. Speech spectrograms of the odd-numbered stimuli are shown in Figure 2.

The 11 stimuli were cross-recorded in random order onto a master tape. Stimuli occurred at 3-sec intervals, and each stimulus occurred 10 times. This tape, consisting of 110 syllables, was used to determine the subjects' phoneme boundary prior to adaptation.

Two adaptation tapes were constructed. These tapes were identical except that the adapting syllable on one tape was $\left[\mathrm{k}^{\mathrm{h}_{\mathrm{a}}}\right]$ (Stimulus 1), while the adapting syllable on the other tape was [ph $\mathrm{p}_{\mathrm{a}}$ (Stimulus 11). These tapes consisted of 10 adaptation trials. Each adaptation trial consisted of 180 presentations of the adapting stimulus (three repetitions every $2 \mathrm{sec}$ for $2 \mathrm{~min}$ ), followed by a 4-sec pause, followed by 11 randomly presented syllables from the test series at 3 -sec intervals. Each test syllable was therefore presented a total of 10 times by the end of the experiment following repeated listening to the adapting syllable. The adapting syllable and the test syllables were recorded at the same amplitude, and all stimuli were presented at a comfortable listening level.

Procedure. Subjects were seated facing a Dynaco loudspeaker in a small room with the experimenter. They were told that they would hear a series of syllables for identification as either " $k$ " or "p." Subjects were told to write " $k$ " or " $p$ " on their answer sheet on every trial. On each day, the subjects first heard the 110 syllables for identification as " $k$ " or "p." Immediately after identifying the 110 syllables, the subjects were presented with the adaptation triais. Half of the subjects, were presented with $\left[\mathrm{k}_{\mathrm{a}}\right]$ as the adapting syllable on Day 1, while half of the subjects heard [pha]. The subjects were told that following the repeated sound there would be a short period of silence, followed by 11 syllables to be identified as "k" or "p."

The procedure in the second session was identical to that of the first except that subjects heard the other adapting stimulus. Thus, each subject was again presented with the 110 syllables for identification, followed by 10 adaptation trials.
Figure 2. Speech spectrograms of every second syllable along a $\left[k^{h_{a}}\right] \cdot\left[p^{\left.h_{a}\right]}\right.$ continuum. Note the decrease in the burst duration preceding the vowel formants as the syllables progress from $\left[k^{h_{a}}\right.$ to $\left[p^{\left.h_{a}\right] \text {. }}\right.$

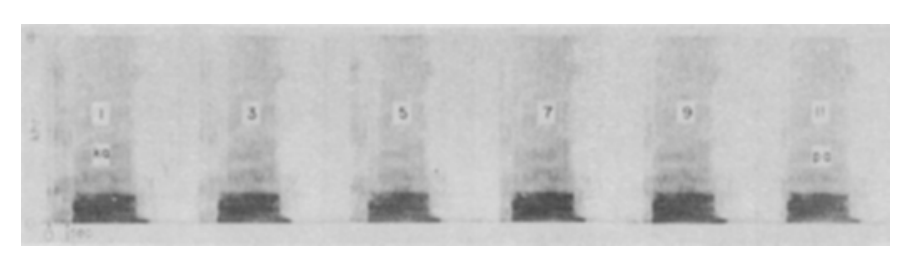




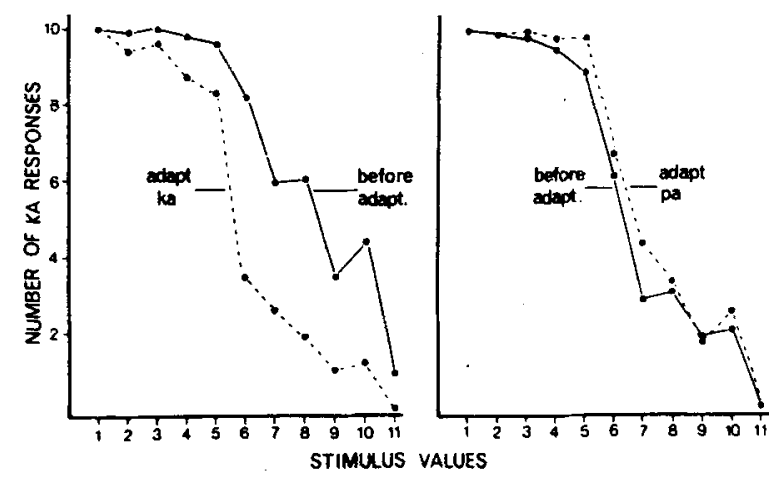

Figure 3. Mean number of $[\mathrm{ka}]$ responses to each stimulus along the $\left[k^{h_{a}}\right]-\left[p^{h_{a}}\right]$ continuum before and after repeated presentation of [ka] (left panel) and [pa] (right panel).

\section{Results}

Figure 3 displays the number of $[k]$ responses for each stimulus before and after adaptation with $\left[k^{h_{a}}\right]$ and $\left[\mathrm{p}^{\left.\mathrm{h}_{\mathrm{a}}\right]}\right.$. It is evident, first, that subjects produce differential responses to the different stimuli in the series. Prior to adaptation, Stimuli 1 through 5 were labeled $[k]$ by all subjects. There is then a decline in [k] responses from about $70 \%$ at Stimulus 6 (averaging over both days for all subjects) to about $25 \%$ at Stimulus 9 . Subjects produced [p] responses to Stimulus $1195 \%$ of the time. The [k]-[p] boundary is thus biased slightly toward the [p] end of the continuum, so that subjects produced more [k] responses overall. Averaging over the 2 days, the $50 \%$ crossover point occurs just to the right of Stimulus 7.

Although most subjects heard a distinct $[p]$ for the later syllables in the series, some subjects reported a slight [k] quality for all syllables. This appears to be a property of the particular syllable that was used to generate the series, since we have produced other series in which this [k] quality was absent.

It should also be noted that the phoneme boundary observed in Figure 3 is less sharply defined than boundaries usually observed in experiments using synthetic stop consonants. However, it should be noted that many of the individual subjects had sharp phoneme boundaries where perception shifted from $[\mathrm{k}]$ to $[\mathrm{p}]$ within a range of two stimulus values, as shown in Figures 4 and 5 . The more gradual slope observed in Figure 3 is partially due to the fact that data are averaged over subjects, and individual subjects shift from $[\mathrm{k}]$ to $[\mathrm{p}]$ at different stimulus values.

Figure 3 reveals a strong effect when $\left[k h_{a}\right]$ was the adapting syllable and a weaker effect for [pha], both in the predicted direction. Thus, adaptation with $\left[\mathrm{k} \mathrm{h}_{\mathrm{a}}\right]$ produced fewer $[\mathrm{k}]$ responses, while adaptation with [pha] produced fewer [p] responses.

The individual and mean areas beneath the curve in Figures 4 and 5 were calculated before and after repeated presentation of $\left[\mathrm{k}_{\mathrm{a}}\right]$ and $\left[\mathrm{ph}_{\mathrm{a}}\right]$ for each subject in each session, and the results of this analysis are presented in Table 1 . Table 1 also displays the difference in the amount of adaptation for the two adapting stimuli. ${ }^{2}$ The results indicate that there were significant shifts in the predicted direction for both $\left[\mathrm{k}_{\mathrm{a}}\right]$ and $\left[\mathrm{ph}_{\mathrm{a}}\right]$ as the adapting stimuli $(\mathrm{p}<.01$ in each instance, one-tailed $t$ test).

It was noticed that the shift in the phoneme boundary in the direction of the adapting syllable was greater for $\left[k^{h_{a}}\right]$ than for $\left[p^{h_{a}}\right]$ for almost all subjects. and this is clearly observed in Figures 4 and 5. Therefore, it was decided to compare the amount of

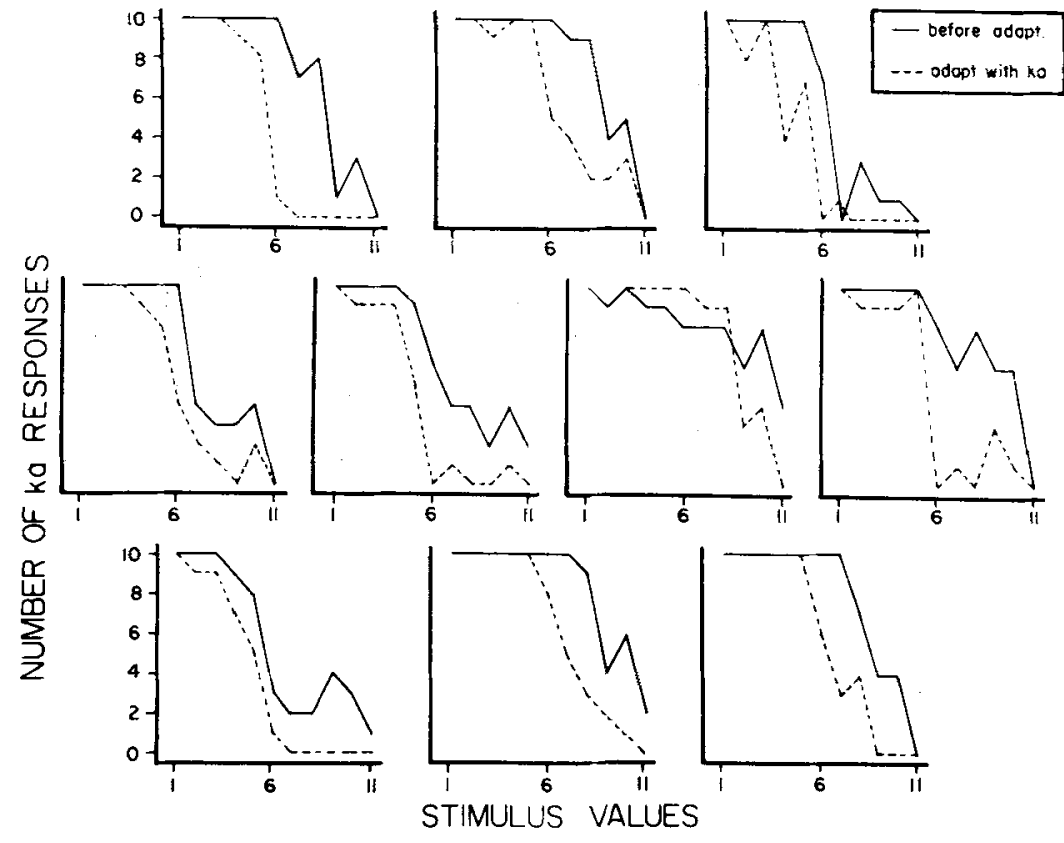

Figure 4. Individual subject's responses to each stimulus in the $\left[k h_{\mathbf{a}}\right]-\left[\mathrm{p}_{\mathrm{a}} \mathrm{h}_{\mathrm{a}}\right]$ series before and after adaptation with $\left[k h_{a}\right]$. 
Figure 5. Individual subject's responses to each stimulus in the $\left[k h_{\mathfrak{a}}\right]-\left[\mathbf{p}_{\mathbf{a}} \mathbf{h}_{\mathbf{a}}\right.$ series before and after adaptation with $\left[p^{h_{a}}\right.$ ].

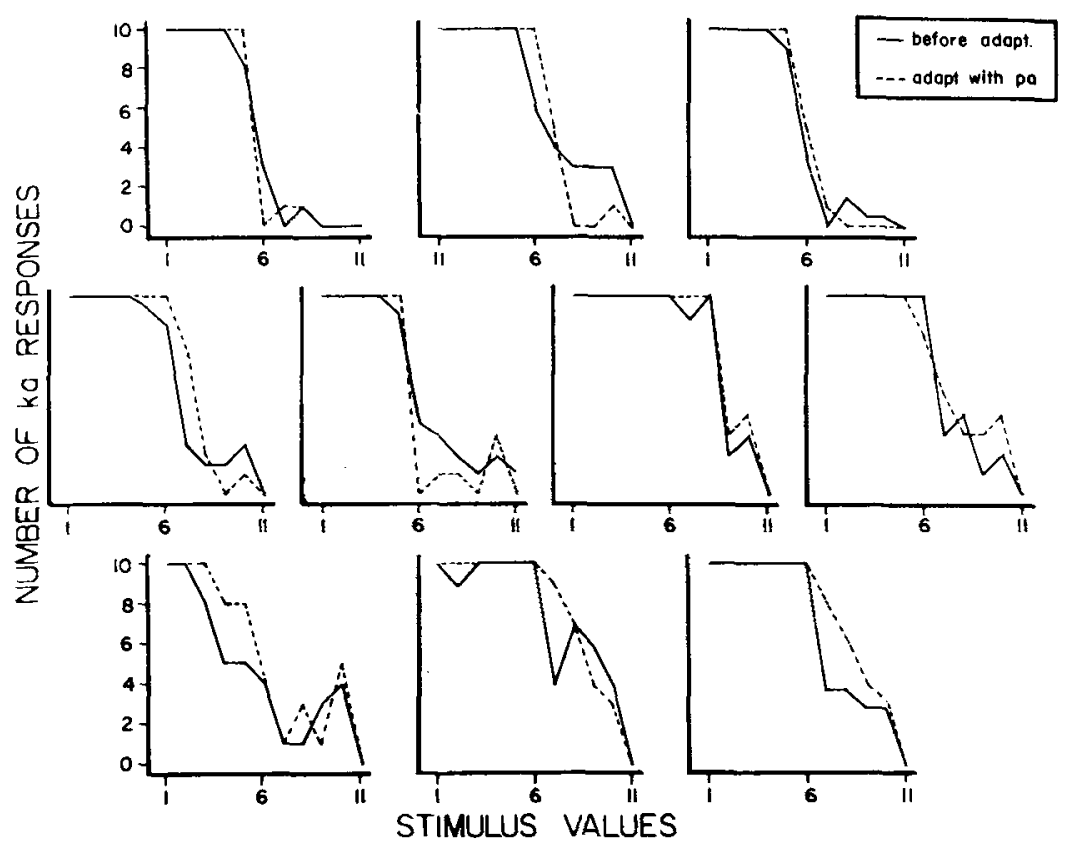

adaptation for the different adapting syllables. Using a correlated $t$ test, it was found that the change was significantly greater for $\left[\mathrm{kh}_{\mathrm{a}}\right]$ adaptation $(\mathrm{p}<.01$, two-tailed $t$ test). ${ }^{3}$ It was also noted, however, that the phoneme boundary was almost always closer to the [pha] than the $\left[\mathrm{k}_{\mathrm{a}}\right.$ ] end of the continuum. This means that there was more room for change in the predicted direction when $\left[\mathrm{k} \mathrm{h}_{\mathrm{a}}\right]$ was the repeated syllable. To insure that the greater change following $\left[\mathrm{k} \mathrm{h}_{\mathrm{a}}\right]$ adaptation was not merely a result of the greater room for change, the following statistical manipulation was employed. First, we computed the amount of change possible in the predicted direction for each subject in each adaptation condition. We then subtracted the possible change for $\left[\mathrm{ph}_{\mathrm{a}}\right]$ adaptation from the possible change for $\left[\mathrm{k}_{\mathrm{a}}\right]$ adaptation. We then computed the actual difference in change between the two conditions for each subject. We were then left with two numbers for each subject, one representing the difference in possible change, the other representing the difference in actual change. If the difference in actual change is merely a function of the difference in possible change, the two would be expected to be highly correlated. In fact, the correlation is -.06 . It thus appears that the difference in adaptation is not merely an artifact of the position of the original boundary.

Although most of our subjects produced a clear phoneme boundary, six subjects did not. These subjects identified almost all of the stimuli as [k], even those at the extreme end of the series. These subjects obviously could not produce more [k] responses following adaptation with $\left[\mathrm{ph}_{\mathrm{a}}\right]$, so their results were not included in Figure 3. However, it is interesting to note that these subjects still produced a significant decrease in $[k]$ responses following adaptation with

Table 1

Area Under the Curve of [ka] Identification

\begin{tabular}{|c|c|c|c|c|c|c|c|}
\hline \multirow[b]{2}{*}{ Subject } & \multicolumn{3}{|c|}{ [ka] Adaptation } & \multicolumn{3}{|c|}{ [pa] Adaptation } & \multirow{2}{*}{$\begin{array}{c}\text { Change } 1 \\
\text { and } \\
\text { Change } 2\end{array}$} \\
\hline & $\begin{array}{c}\text { Pre- } \\
\text { Adaptation }\end{array}$ & $\begin{array}{c}\text { Post- } \\
\text { Adaptation }\end{array}$ & Change 1 & $\begin{array}{c}\text { Pre- } \\
\text { Adaptation }\end{array}$ & $\begin{array}{c}\text { Post- } \\
\text { Adaptation }\end{array}$ & Change 2 & \\
\hline 1 & 74 & 42 & -30 & 47 & 47 & 0 & -30 \\
\hline 2 & 82 & 60 & -22 & 64 & 61 & -3 & -25 \\
\hline 3 & 57 & 35 & -22 & 43 & 51 & 8 & -14 \\
\hline 4 & 69 & 51 & -18 & 53 & 66 & 13 & -5 \\
\hline 5 & 65 & 38 & -27 & 47 & 50 & 3 & -24 \\
\hline 6 & 78 & 79 & 1 & 79 & 82 & 3 & 4 \\
\hline 7 & 79 & 47 & -32 & 65 & 68 & 3 & -29 \\
\hline 8 & 56.5 & 36 & -20.5 & 46 & 56 & 10 & -10.5 \\
\hline 9 & 85 & 64 & -21 & 75 & 78 & 3 & -18 \\
\hline 10 & 80 & 58 & -22 & 69 & 76 & 7 & -15 \\
\hline Mean & 72.55 & 51.2 & -21.35 & 58.8 & 63.5 & 4.7 & -16.65 \\
\hline
\end{tabular}




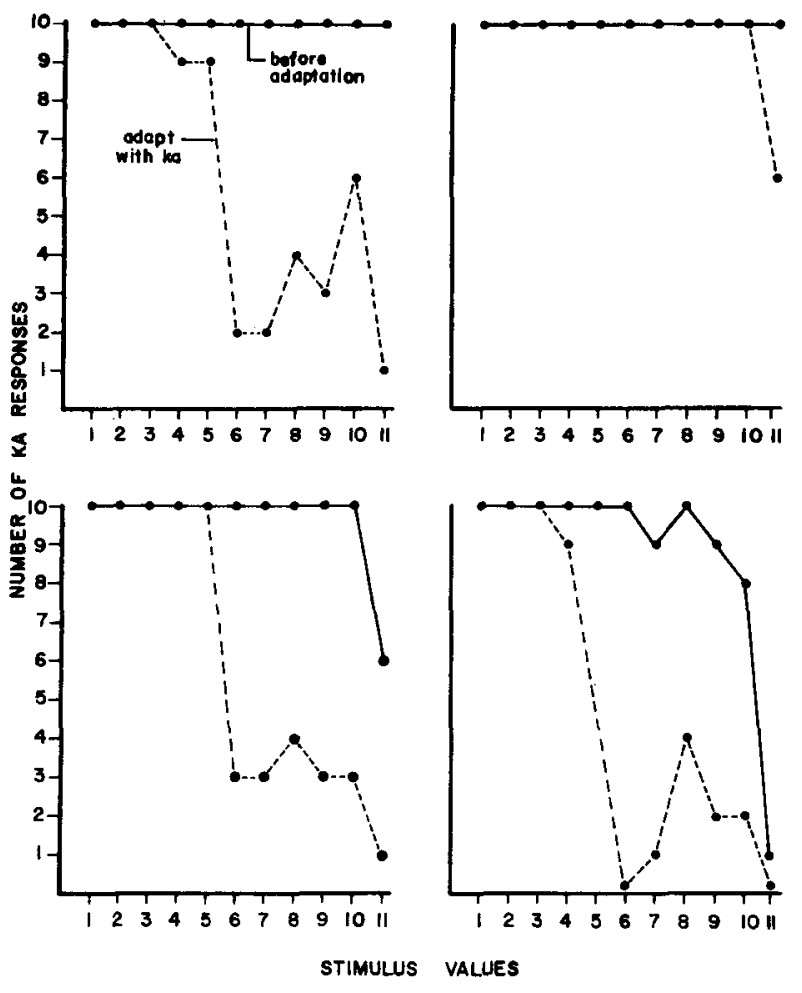

Figure 6. Number of [ka] responses to each stimulus in the

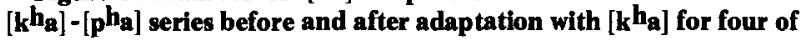
the six subjects who do not show an appropriate phoneme boundary. Note that the subject whose data are displayed on the bottom right panel did produce a sharp phoneme boundary. This boundary was so far to the right, however, as to preclude a shift in the predicted direction following adaptation with $\left[p^{h_{a}}\right]$.

[ $\mathrm{k}_{\mathrm{a}}$ ]. This result is displayed in Figure 6 for four of the subjects. It can be seen that several of these subjects did not identify a single stimulus in the original test series as [p]. Following adaptation with [ $\mathrm{k}$ ha], there was a dramatic increase in the number of [p] responses for stimuli at the end of the series [i.e., those on the right of the graph). It thus appears that adaptation can be observed without first producing a phoneme boundary.

\section{Discussion}

Experiment I demonstrates that selective adaptation can occur with a series bridging two stop consonants previously thought to be nonoverlapping. Repeated presentation of [ $\left.\mathrm{k} \mathrm{h}_{\mathrm{a}}\right]$ shifts the boundary of a $[k]-[p]$ series toward $[k]$, while repeated presentation of $\left[\mathrm{ph}_{\mathrm{a}}\right]$ shifts the boundary toward $[\mathrm{p}]$.
It was also found that $\left[k_{a}\right]$ is a more effective adapting stimulus than [ $\mathrm{ph}_{\mathrm{a}}$ ], since there is a larger shift in the boundary of the $[\mathrm{k}]-[\mathrm{p}]$ series following adaptation with $\left[\mathrm{k}_{\mathrm{a}}\right]$ than with $\left[\mathrm{ph}_{\mathrm{a}}\right]$.

It is not possible to specify a single acoustic feature which was adapted in this particular experiment. As Cole and Scott (1974a) and Scott (1974) have pointed out, [k] and [p] are probably the most acoustically complex consonants in English. Both $[\mathrm{k}]$ and $[\mathrm{p}]$ in syllable initial position are cued by at least three different types of acoustic features-the frequency of the stop burst and aspiration; transitions which are embedded within the aspiration; and the shape of the waveform itself, i.e., its envelope. These different cues interact in complex ways, since they contribute differentially to perception with different vowels (Scott, 1974; Fisher-Jørgenson, Note 2).

Because of the interaction of cues at the level of phoneme identification, it is difficult to determine the precise manner in which these cues are affected by splicing successively longer segments from the original $\left[\mathbf{k}{ }_{a}\right]$. For example, splicing into $\left[k^{h_{a}}\right.$ ] first removes the 8-15-msec stop burst for [k], shortens and/or changes the starting frequency of any transitions, and simultaneously affects the shape of the waveform envelope, and all of these features are important for the $[\mathrm{k}]$ - $[\mathrm{p}]$ discrimination (Scott, 1974).

\section{EXPERIMENT II}

Fortunately, not all series which can be constructed by splicing into a CV syllable are as difficult to describe acoustically as the $[k]-[p]$ series. The series used in Experiment II, which was constructed by splicing successively longer segments from the onset of [ja] (as in "job"), produces an almost ideal series from [ja] to [da]. Both [j] and [d] are preceded in ongoing speech by a closure interval (realized acoustically as a short silent interval) and are released with an explosive burst, so that both have an abrupt onset. Thus, onset abruptness is not much affected by splicing into [j]. Moreover, the vowel transitions for a naturally spoken [ja] and [da] are nearly identical. Finally, examination of speech spectrograms reveals that the frequency composition of the consonant noise is quite similar for a naturally spoken [ja] and [da]. Compare, for example, the speech spectrograms of the naturally spoken [da] in Figure 1 and the final two stimuli in Figure 7, which were constructed by splicing into [ja] (and were heard by all subjects as

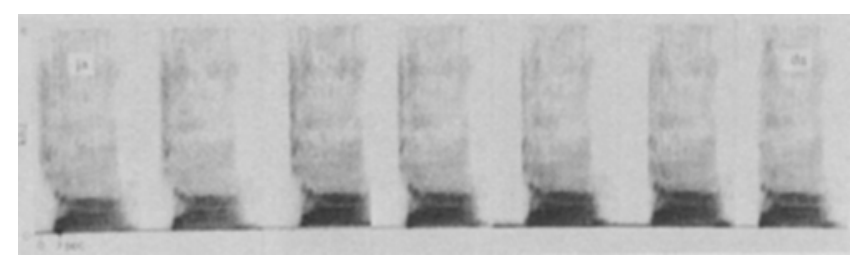

Figure 7. Speech spectrograms of syllables produced by splicing 10 -msec segments from the onset of the stimulus displayed at the far left of the figure. 
[da]). Thus, a series from [ja] to [da] presents a continuum which differs mainly in the duration of the burst prior to the vowel transitions.

\section{Method}

Subjects. Twelve students from an introductory psychology course served as subjects. Each subject was paid $\$ 2$ for his participation in two $1 / 2$-h sessions 2 days apart. Subjects were tested in groups of three in a sound-attenuated room.

Stimuli. Stimuli were the seven CV syllables displayed in Figure 7. These stimuli were constructed by recording a naturally spoken [ja] produced by the same speaker as in Experiment I (who served as informant in all further experiments). The duration of the consonant noise prior to the onset of the vowel transitions was $70 \mathrm{msec}$, and the duration of the transition-vowel unit was approximately $275 \mathrm{msec}$. The original [ja] was cross-recorded a number of times and the series of seven syllables was constructed by splicing $0,10,20,30,40,50$, or $60 \mathrm{msec}$ into the syllable. Thus, Stimulus 1 , displayed at the far left of Figure 7 , contains $70 \mathrm{msec}$ of consonant noise prior to the vowel transitions, while Stimulus 7 at the far right of Figure 7 contains $10 \mathrm{msec}$ of consonant noise prior to the vowel transitions.

Two stimulus tapes were constructed. These tapes were identical, except that on one tape Stimulus 1 ([ja]) was used as the adapting syllable while Stimulus 7 ([da]) was used on the other. Each tape consisted of 70 randomly ordered syllables spaced $3 \mathrm{sec}$ apart. Each of the seven stimuli were thus presented 10 times for identification as either [ja] or [da].

The identitication test was followed shortly after by seven adaptation trials. Each adaptation trial consisted of exactly 2 min of listening to the adapting stimulus (either [ja] or [da], depending upon the day) presented at a rate of three presentations every $2 \mathrm{sec}$, for a total of 180 presentations. The adapting stimulus was followed by a $3-\mathrm{sec}$ pause, followed by 10 randomly presented stimuli from the test series for identification as either [ja] or [da]. Thus, each stimulus in Figure 7 was presented 10 times for identification before adaptation and 10 times following adaptation with [ja] or [da].

Procedure. Half of the subjects were presented with [ja] as the adapting stimulus during the first session, and [da] as the adapting stimulus during the second; the order was reversed for the remaining subjects. In all respects, the procedure was identical to the previous experiment, except that subjects were instructed to write either " $\mathrm{j}$ " or " $\mathrm{d}$ " after hearing each syllable.

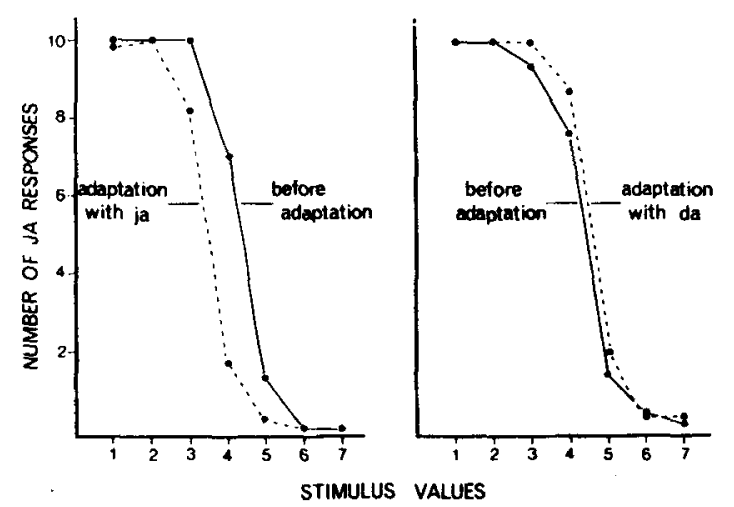

Figure 8. Mean number of [ja] responses to stimuli along the [ja]-[da] continuum before and after repeated presentation of [ja] or [da].

\section{Results}

The results of the experiment are displayed in Figure 8. As in Experiment I, correlated t tests were used to compare the area under the curve before and after repeated presentation with [ja] or [da]. A significant change in the predicted direction was found for both [ja] and [da] ( $\mathrm{p}<.01$, in both cases). Thus, compared to the preadaptation test series, there were fewer [j] responses following repeated presentation of [ja], and fewer [d] responses following repeated presentation of [da]. It is clear from Figure 8 that adaptation is selective, in that the change in the subjects' responses occurred only for those stimuli near the phoneme boundary.

A dramatic result of the present experiment is that the amount of change following adaptation with [ja] is greater than that following adaptation with [da] $(p<.01)$. The consistency of this asymmetrical adaptation effect may be seen by comparing Figures 9 and 10 , which display the amount of adaptation for
Figure 9. Individual subject's responses to stimuli along the [ja]-[da] continuum following repeated presentation of [ja].
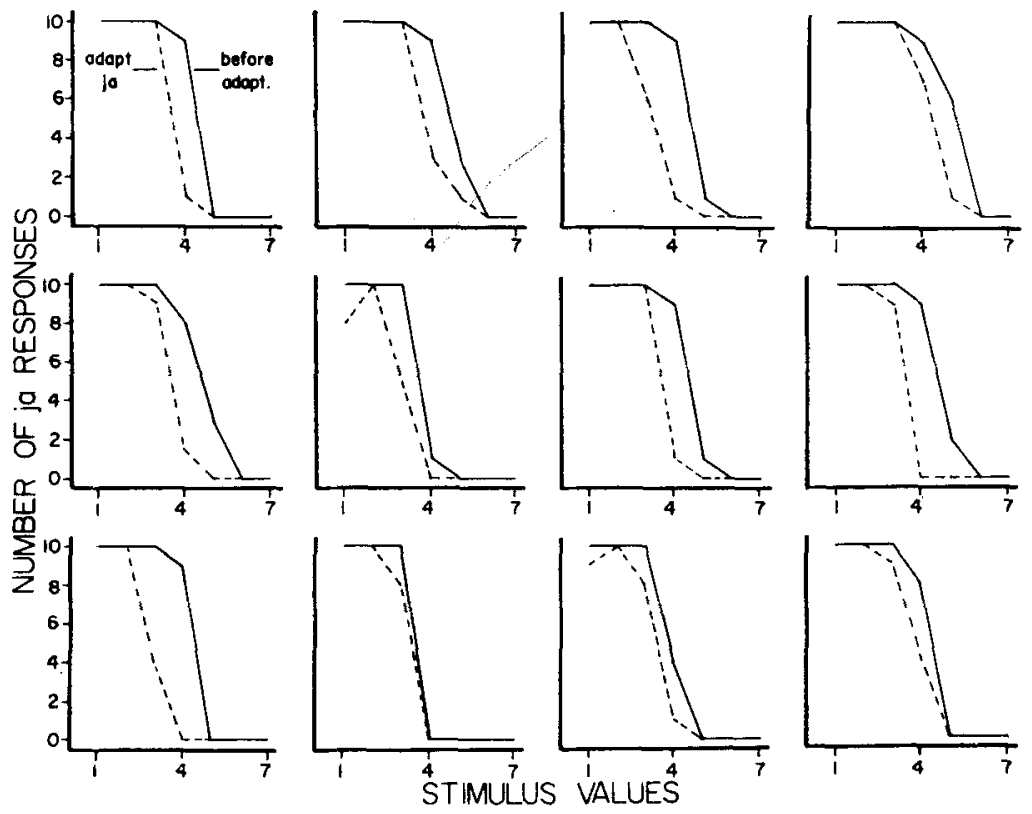

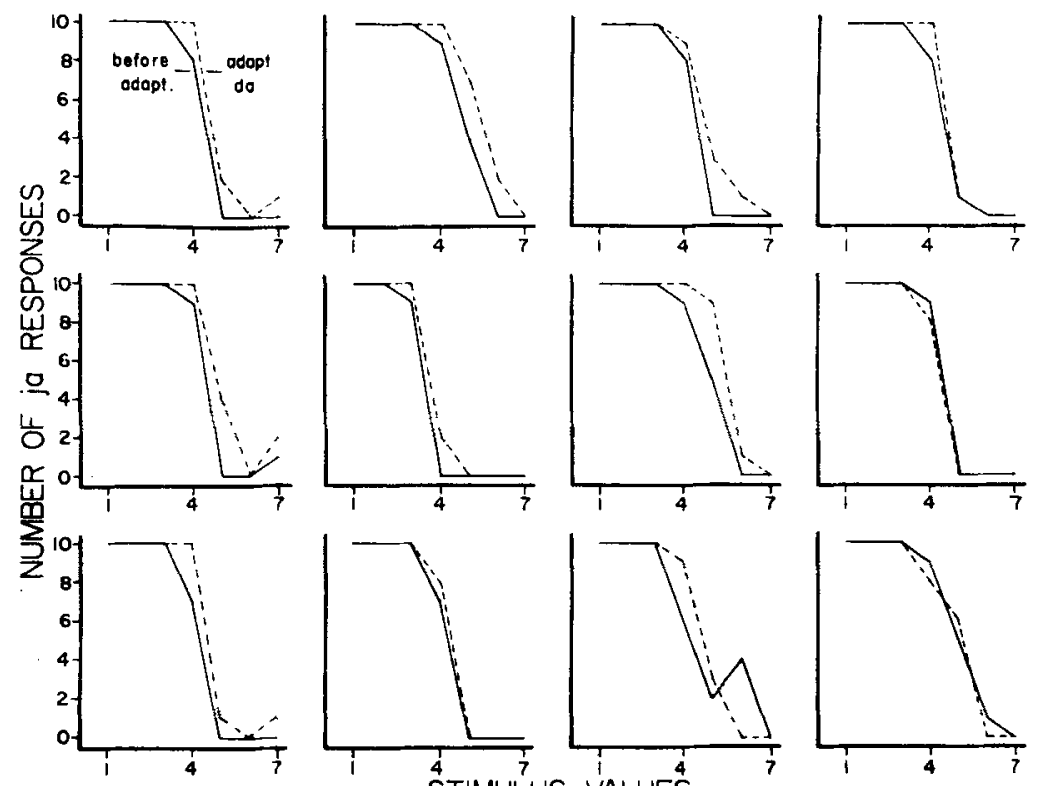
Figure 10. Individual subject's responses to stimuli along the [ja]-[da] continuum following repeated presentation of [da].
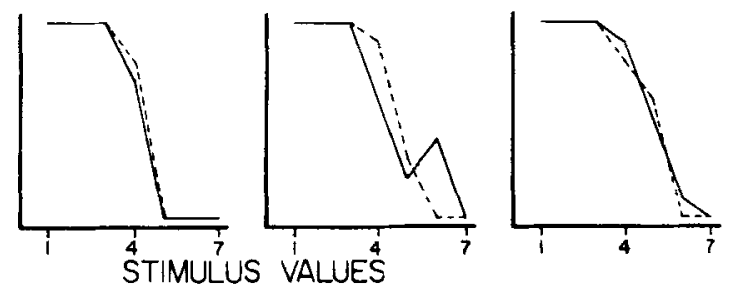

each subject after repeated listening to [ja] and [da], respectively. Figure 9 shows that all subjects produced a large (average about one stimulus value) shift in the phoneme boundary in the predicted direction following repeated presentation of [ja], while 9 of 12 subjects produced a shift in the phoneme boundary in the opposite direction following repeated presentation of [da]. A comparison of the magnitude of the boundary shift for the individual subjects in Figures 9 and 10 reveals that all 12 subjects exhibit a greater shift in the phoneme boundary following adaptation with [ja]. These figures also reveal the remarkable consistency with which subjects identified each stimulus as either [j] or [d].

\section{Discussion}

Experiment II provides clear evidence for selective adaptation, and reveals an interesting phenomenon which we have called asymmetrical adaptation. Eimas and Corbit (1973) observed a similar difference in the magnitude of boundary shift along a voiced-voiceless continuum for a voiced vs. voiceless adapter. In the present experiment, asymmetrical adaptation can be accounted for on the basis of the acoustic composition of the stimuli. Considering the nature of the series of syllables used in this experiment, it is clear from Figure 7 that the major acoustic feature for distinguishing between [j] and [d] is the duration of the frication prior to the vowel transitions. If the 70-msec consonant noise from Stimulus I in Figure 7 is spliced onto a steady-state [a], a perfectly natural [ja] is heard. If the $10-\mathrm{msec}$ burst from Stimulus 7 is spliced onto a steady-state vowel [a], a natural [da] is heard (Cole \& Scott, 1974b).

This simple demonstration illustrates that the duration of the burst is the main feature which determines perception of [ja] or [da]. We may assume that analyzers exist in the auditory system which respond maximally to energy with certain spectral and durational characteristics. The consonants [j] and [d] have virtually identical onset and spectral characteristics, and differ only in the duration of the fricative noise. Thus, perception of [j] and [d] may be mediated by separate analyzers, each maximally responsive to a particular duration of noise. Repeated presentation of [ja] or [da] is assumed to reduce the responsiveness of the appropriate analyzer, resulting in a decrease in categorization of test syllables belonging to the same phonetic category as the adapting syllable. In order to explain asymmetrical adaptation effects. a binary system composed of a long-and short-burst analyzer requires an additional post hoc assumption. It must be assumed that the short-burst detector is less affected by adaptation than the long-burst detector.

Alternately, a single analyzer may exist which responds maximally to [j] and with reduced vigor to [d]. This analyzer would therefore be affected to a much greater extent by the [ja] adapter than the [da] adapter. ${ }^{4}$

\section{EXPERIMENT III}

Both of the models described above assume that adaptation of [j] and [d] is determined by analyzers sensitive to the duration of the fricative noise prior to the vowel transitions. It is also possible, however, that vowel transitions may interact with burst duration as a cue for the [j]-[d] discrimination. At intermediate burst durations, the presence of appropriate vowel transitions may bias perception toward [da]. With adaptation of these transitions, perception would then 
be shifted to [ja], resulting in the predicted adaptation effect.

Experiment III examines the influence of vowel transitions on the [ja]-[da] distinction by using the syllables [ji] and [di] as adapting stimuli with the [ja]-[da] test series employed in Experiment II. The transitions for a naturally spoken [ji] and [di] are not nearly as pronounced as the transitions for [ja] and [da], but more importantly, the second formant transition for [ji] and [di] rises to the second vowel formant, and is thus acoustically dissimilar to the falling second formant transition observed in [ja] and [da]. Since repeated presentation of [di] should not adapt the transition detectors for stimuli along the [ja]-[da] test series, a positive result of adaptation with [di] would suggest that adaptation of [d] does not depend upon adaptation of transitions. Repeated presentation of [ji], however, should produce adaptation effects comparable to those observed in Experiment II, since [ji] shares the same duration of consonant noise as [ja].

\section{Method}

Subjects. Twenty-four students from the University of Waterioo served as subjects. Each subject was paid $\$ 2$ for participation in a single $1 / 2-h$ session. Half of the subjects were presented with [jil as the repeated syllable, the other half were presented with [di].

Stimuli. All stimulus conditions were identical in Experiments II and III. except that a naturally spoken [ji] and a naturally spoken [di] were used as the adapting stimuli. The test stimuli in the initial identification task and following repeated presentation of [ji] and [di] were drawn from the [ja]-[da] series. III.

Procedure. The procedure was identical in Experiments II and

\section{Results}

Figure 11 displays the effect of repeated presentation of [ji] and [di] on the [ja]-[da] boundary. The effect of repeated presentation of [ii] and [di] was nearly identical in magnitude to the shifts produced by [ja] and [da]. Correlated $t$ tests based on the area under the curve before and after repeated presentation of [ji] and [di] revealed a significant adaptation effect for both syllables $(p<.01)$, and a significantly greater shift in the phoneme boundary following adaptation with [ji].

\section{EXPERIMENT IV}

All demonstrations of selective adaptation to date have employed a single methodology, in which subjects identify syllables along some continuum before and after listening to an adapting stimulus. The present experiment attempts to demonstrate selective adaptation using a different experimental task-choice reaction time.

The rationale for Experiment IV is based on a finding by Pisoni and Tash (1974; see their Figure 3)-that choice reaction time to syllables along a VOT continuum is longest for syllables at or near

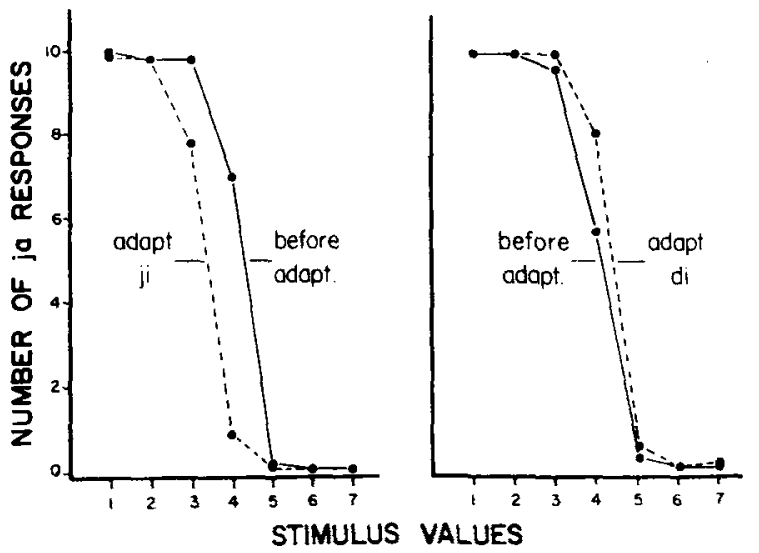

Figure 11. Mean numberr of [ja] responses to stimuli along the [ja]-[da] continuum before and after repeated presentation of a naturally spoken $[j i]$ and $[d i]$.

the phoneme boundary. Generalizing to our stimuli, this means that subjects presented with syllables from the [ja]-[da] continuum should take longest to classify Syllable 5 as either [ja]-[da], since this syllable represents the phoneme boundary. Since repeated presentation of [ja] results in a clear shift in the phoneme boundary from Syllable 5 to Syllable 4, we should also find a corresponding shift in the peak of the choice reaction time function following adaptation.

\section{Method}

Subjects. Fifteen students from Carnegie-Mellon University served as subjects. Each subject was tested individually in a small sound-attenuated room in a session lasting approximately $45 \mathrm{~min}$.

Stimuli. The same stimulus tape was used as in the previous experiment.

Procedure. Subjects sat facing a loudspeaker connected to a Sony TC 630 tape recorder. The output of the tape recorder was also connected to a voice-operated relay, which was triggered by the onset of each syllable. The voice-operated relay was used to start a Hunter millisecond timer, which was stopped when the subject pushed a response button.

Each subject sat with the second and fourth fingers of his right hand resting on a microswitch, labeled "JA" and "DA." The particular response required from each finger was counterbalanced across subjects. The subject was instructed to respond as quickly as possible after hearing each syllable by pushing the appropriate response key, and to inform the experimenter if he made an unintended response. On each trial, the experimenter recorded the subject's response ([ja] or [da]) and reaction time, and reset the timer. The experiment was preceded by 10 practice trials.

\section{Results and Discussion}

The main results of the experiment are presented in Figure 12.5 The bottom panel in Figure 12 displays the mean number of [ja] responses to each stimulus before and after adaptation with [ja]. There is a clear shift in the phoneme boundary toward the adapting syllable following repeated presentation of [ja], as demonstrated in the previous two experiments ( $p<.001$, based on area under the curve).

The top panel of Figure 12 displays the choice reaction time to each syllable before and after 

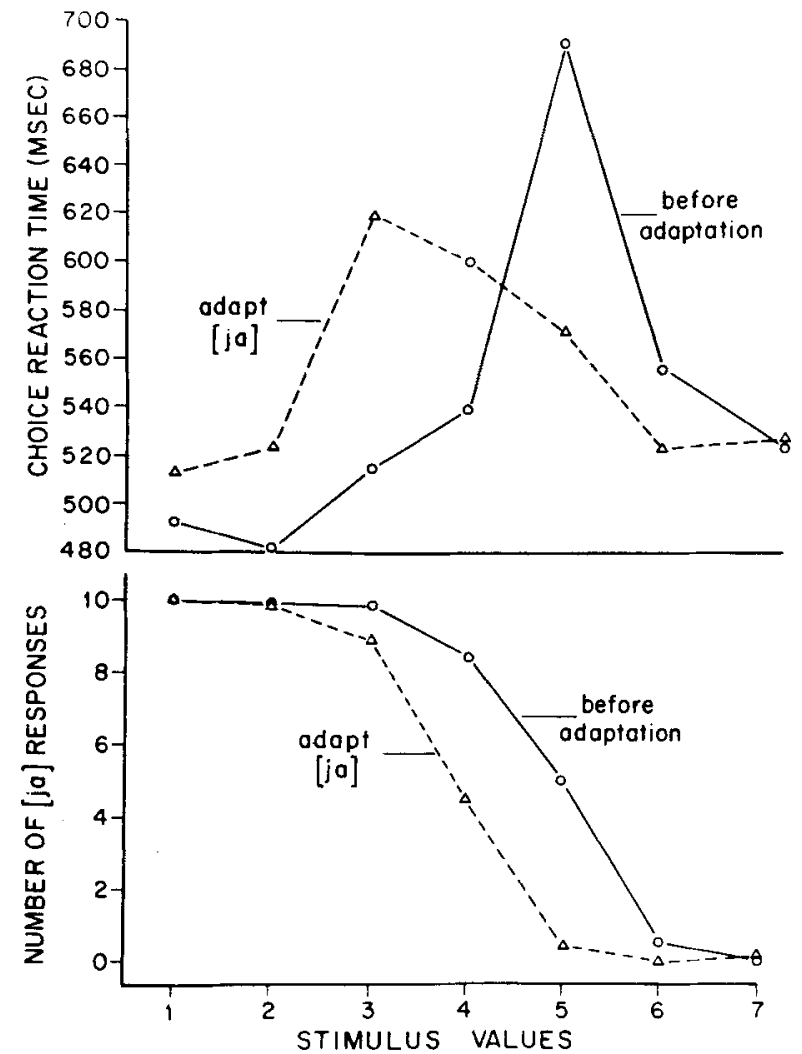

Figure 12. Top panel: Choice reaction time to each syllable in the [ja]-[da] series before and after adaptation with [ja]. Each point is based on an average of the median reaction time scores to each syllable. Bottom panel: Mean number of [ja] responses to each syllable before and after adaptation.

adaptation with [ja]. It is apparent, first, that, prior to adaptation. reaction time increases as syllables change from an unambiguous [ja] to a syllable which is ambiguous.
A comparison of the upper and lower panels in Figure 12 reveals that the peak in the reaction-time function before adaptation occurs precisely at the category boundary between [ja] and [da]. Following adaptation with [ja], there is a shift in the peak of the reaction time function in the predicted direction. Analysis of variance, computed on the median reaction time (RT) score to each syllable before and after adaptation, revealed a significant difference in the RTs to the different stimuli $(F=4.2 . \mathrm{df}=6 / 84$, $p<.001)$ and a signiticant effect of the adapting stimulus on reaction times to the different stimuli. This later effect occurs as an interaction between syllable position and the pre- vs. postadaptation scores $(F=6.68$. df $=6 / 84, p<.001)$. A comparison of the dashed lines in the top and bottom panels in Figure 12 reveals that the shift in the peak of the RT function following adaptation with [ja] is greater than the corresponding shift in the phoneme boundary based on the identification responses.

The identification and RT data for the individual subjects are displayed in Figures 13 and 14, respectively. Examination of Figure 13 reveals that individual subjects identified the different stimuli with great consistency-all subjects produced a sharp phoneme boundary which shifts in the predicted direction following adaptation with [ja]. The choice RT data for the individual subjects are less elegant, although a clearly discernible shift in the distribution of the RT scores in the predicted direction may be observed for 11 out of 15 subjects, and a clear shift in the peak of the function may be seen for 9 subjects. Inspection of Figure 14 reveals that, as with the identification responses, the postadaptation shift in the individual subject's RTs was restricted to the region of the phoneme boundary. That is, following adaptation, there was no significant increase in RT

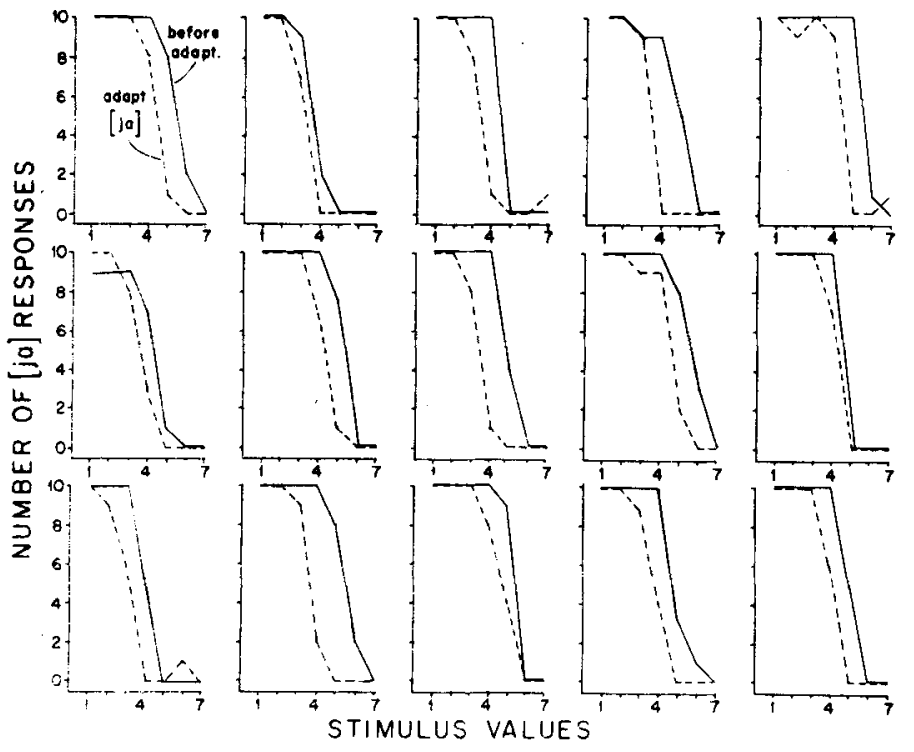

Figure 13. Individual subject's identification responses to stimuli along the [ja]-[da] series before and after adaptation with [ja]. 
Figure 14. Individual subject's choice rection times to each syllable along the [ja]-[da] series before and after adaptation with [Ja].

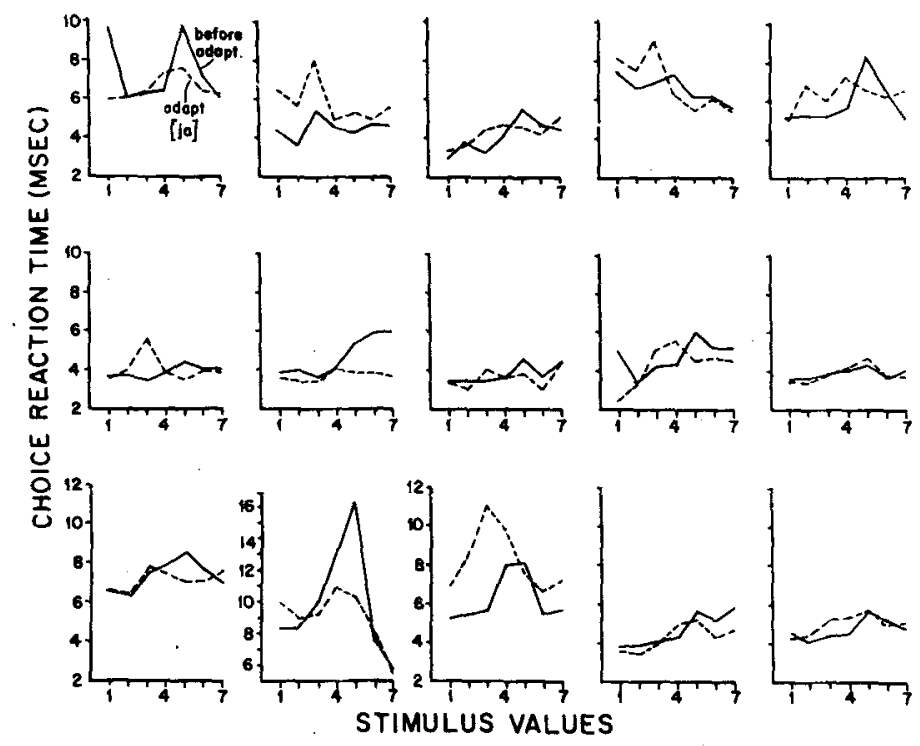

following adaptation for the first syllable in the series.

The RT data presented in Figures 12 and 14 do not differentiate between [ja] and [da] responses. Each point in these figures reflects the time it took subjects to decide if the stimulus was either [ja] or [da] and the RTs were combined for the two responses. The RT data for the [ja] and [da] responses to each syllable are presented in Table 2. Note that only Syllable 4 provides data for both [ja] and [da] responses before and after adaptation with [ja]. Because of the sharp phoneme boundaries, there were virtually no [da] responses at Syllable 3 before adaptation and no [ja] responses at Syllable 5 following adaptation. The RTs in Table 2 are consistent with the combined data presented in Figure 12. Before adaptation, the longest reaction times occur at Syllable 5 for both [ja] and [da], and this syllable was identified equally often as [ja] and [da]. Following adaptation, Syllable 5 was identified by all subjects as [da], and there is a corresponding decrease in the RT for [da] responses, from 710 to $580 \mathrm{msec}$. At Syllable 4, [da] responses fell from about $50 \%$ to $15 \%$ following adaptation, and there was virtually no change in RT for [da] responses. However, [ja] responses to Syllable 4 fell from $85 \%$ to $50 \%$ following adaptation, and there was a corresponding rise in the RT to [ja] from $526 \mathrm{msec}$ before adaptation to $725 \mathrm{msec}$ following adaptation. Thus, for Syllable 4, there was a large pre- and postadaptation difference in RT to [ja], but not to [da]. For Syllable 3, the RTs for [ja] responses are again consistent with the identification responses. Identitication of [ja] fell from $100 \%$ to about $90 \%$ following adaptation, and RT increased by $112 \mathrm{msec}$.

The results of the present experiment provide additional support for selective adaptation of speech sounds. Repeated presentation of [ja] resulted in a shift in the phoneme boundary of a [ja]-[da] continuum, such that fewer [ja] responses were produced following adaptation. This postadaptation shift in identification responses was accompanied by a corresponding shift in choice RT to the [ja]-[da] syllables. Prior to adaptation with [ja], the peak of the RT function occurred at the phoneme boundary. Following adaptation with [ja], the peak of the RT function shifted in the same direction as the postadaptation phoneme boundary.

The main conclusion that can be drawn from Experiment IV is that choice RT may be used as an additional measure of selective adaptation. In fact, the choice RT procedure may provide an especially efficient tool for studying selective adaptation, since the subject's identification responses before and after adaptation (the traditional measure) can be compared directly with choice $\mathrm{RT}$ responses in the same

Table 2

Mean Reaction Time (in Milliseconds) to Syllables 3, 4, and 5 Before and After Adaptation with [ja]

\begin{tabular}{lccc}
\hline & \multicolumn{2}{c}{ Syllable } \\
\cline { 2 - 3 } & 3 & 4 & 5 \\
\hline & & [da] & 710 \\
Pre & 630 & 580 \\
Post & 630 & 644 & \\
& & {$[j a]$} & 761 \\
Pre & 513 & 526 & \\
Post & 625 & 725 & \\
\hline
\end{tabular}

Note. Mean scores are based on the median reaction time to each syllable for each subject. 


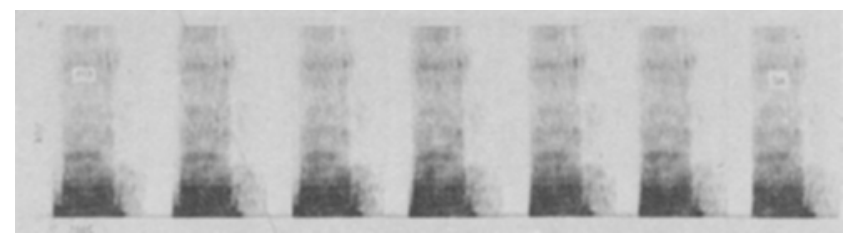

Figure 15. Speech spectrograms of syllables in the [ma]-[ba] series. Note the abrupt onset for the first $[\mathrm{ma}]$ in the series. experiment. The present experiment revealed some interesting differences in the measures. First, the shift in the phoneme boundary following adaptation, based on the identification responses, was extremely consistent for the different subjects, while the shift in the choice RT function showed a great deal more variability (cf. Figures 13 and 14). Second, the overall shift in the choice RT function following adaptation was greater than the corresponding shift in the phoneme boundary based on the identification responses. Unfortunately, because of the greater variability in choice RT across subjects, it would be premature to conclude that $\mathrm{RT}$ is a more sensitive measure of selective adaptation until the present results have been replicated.

\section{EXPERIMENT $V$}

Experiment $\mathrm{V}$ was undertaken to determine if selective adaptation could be observed for the nasal consonant $[\mathrm{m}]$ using a series from [ma] to [ba].

\section{Method}

Subjects. Twelve students from the University of Waterloo served as subjects in two $1 / 2$-h sessions 2 days apart. Each subject received $\$ 2$ for his participation.

Stimuli. The original syllable used to construct the test series was a naturally spoken [ma] with $95 \mathrm{msec}$ of nasal resonance prior to the vowel transitions and $242 \mathrm{msec}$ of transitions and vowel. Pretesting with this stimulus revealed that an ambiguous stimulus (either [ma] or [ba]) was not heard until approximately $60 \mathrm{msec}$ of nasal resonance was removed from the syllable. In order to construct a series of equally spaced stimuli with the phoneme bcundary near the middle of the series, we chose a syllable with $54 \mathrm{msec}$ of nasal resonance (i.e., $41 \mathrm{msec}$ removed from the onset of the original [ma]) as the first syllable of the series. This syllable was identified as [ma] $93 \%$ of the time. The series displayed in Figure 15 was then constructed by splicing $9 \mathrm{msec}$ further into each syllable, so that the final syllable in the series, which was heard as [ba] by all subjects, consisted entirely of vowel transitions followed by steady-state vowel. One advantage of this manipulation was that all syllables in Figure 15 have an abrupt onset, since even the first syllable in the series had been spliced into; a series starting with an unaltered [ma] would have progressed from a gradual to an abrupt onset.

Stimulus tapes were similar to previous experiments. The identification test consisted of the syllables displayed in Figure 15 presented 10 times each in random order, followed by seven adaptation trials with either [ma] (Stimulus 1) or [ba] (Stimulus 7) as the adapting stimulus. As before, 10 syllables were randomly presented for identification following each presentation of the adapting stimulus.

Procedure. The procedure was identical to that of Experiments II and III, except that subjects were told to write " $\mathrm{m}$ " or " $\mathrm{b}$ " for each test syllable.

\section{Results}

The results, displayed in Figure 16, reveal a significant adaptation effect for both [ma] $(p<.01)$ and $[\mathrm{ba}](\mathrm{p}<.01)$ and significantly more adaptation for [ma] than for [ba] $(p<.01)$. Possibly because of the short duration of nasal resonance for stimuli in this series, repeated presentation of [ma] produced a decrease in $[\mathrm{m}]$ responses for all syllables labeled $[\mathrm{m}]$ prior to adaptation, even the first syllable in the series. It is clear, however, that adaptation is greatest for stimuli near the phoneme boundary.

\section{EXPERIMENT VI}

Previous experiments demonstrated selective adaptation with a voiceless stop, affricate, and nasal consonant. In Experiment III, selective adaptation was observed when the adapting syllables ([ji] and [di]) contained a different vowel than syllables in the test series. In all experiments, however, the adapting syllable contained a consonant phoneme that was also present in the test series. Therefore, adaptation could be occurring at the level of phoneme categorization, at a level of abstract feature categorization or at an acoustic level. The present experiment was performed to determine whether selective adaptation would occur along the [ma]-[ba] continuum when a naturally spoken [na] was used as the adapting syllable. If selective adaptation along the [ma]-[ba] continuum occurs at a phonemic level of analysis, then [na]
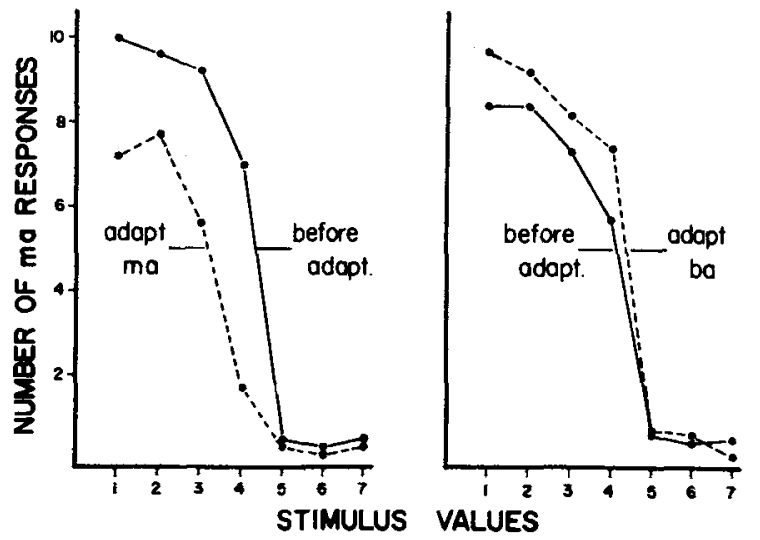

Figure 16. Mean number of [ma] responses to stimuli in the [ma]-[ba] series before and after repeated presentation of [ma] and [ba]. 
should not produce an adaptation effect, since it contains a different nasal phoneme. If adaptation occurs (to whatever extent) at either an abstract or acoustic feature level, then [na] should produce a shift in the phoneme boundary of the [ma]-[ba] series, since the nasality component is perceptually identical for $[m]$ and $[n]$ in CV syllables (Malécot, 1956). The present experiment was performed to test for "crossed-adaptation" effects similar to those found by Eimas and Corbit (1973) along the voiced-voiceless continuum.

\section{Method}

Subjects. Twelve students enrolied in an introductory psychology class at Carnegie.Mellon University served as subjects in a single session lasting approximately $45 \mathrm{~min}$. The subjects were tested in groups of two, three, or four in a small sound-attenuated room.

Stimuli. Syllables from the [ma]-[ba] continuum shown in Figure 15 and described in Experiment $V$ were used as stimuli. The order of presentation of syllables in the identification and adaptation trials was identical in Experiments IV and V; only the adapting syllable differed in the two experiments. The adapting syllable was a naturally spoken [na] with $71 \mathrm{msec}$ of nasal resonance followed by $247 \mathrm{msec}$ of vowel transitions and steady-state vowel. The amplitude of the adapting syllable was matched for the amplitude of the test syllables when the master tape was prepared. The adapting syllable was presented at the rate of three presentations every $2 \mathrm{sec}$ for $2 \mathrm{~min}$.

Procedure. All aspects of the procedure were identical to the previous experiment, with one important exception-all stimuli were presented binaurally over Koss Pro 4AA stereophonic headphones connected to a Sony TC 630 tape recorder. In previous experiments. stimuli were presented over a loudspeaker.

\section{Results}

The results of Experiment VI are shown in Figure 17. A small but significant adaptation effect was observed using one-tailed $t$ tests based on either the area under the curve before and after adaptation or a comparison of the $50 \%$ crossover point (by extrapolation) before and after adaptation ( $p<.02$ for both tests). This later $t$ test was employed since there were slightly more postadaptation [ba] responses to all stimuli, and this pattern affects the area under the curve in the predicted direction, while the $t$ test based on the $50 \%$ crossover point is sensitive only to shifts in the phoneme boundary. It should be noted that the boundary shift is considerably smaller with the [na] adapter than with the [ma] adapter (Figure 16).

\section{EXPERIMENT VII}

Experiment VI revealed a crossed-adaptation effect of a [na] adapting stimulus on a [ma]-[ba] continuum, and indicates that selective adaptation occurs at least partially at the level of an acoustic or phonetic feature for nasality. Experiment VII was designed to test for selective adaptation along the [ma]-[ba] continuum following repeated presentation of the nasal resonance excised from its syllable context and presented in

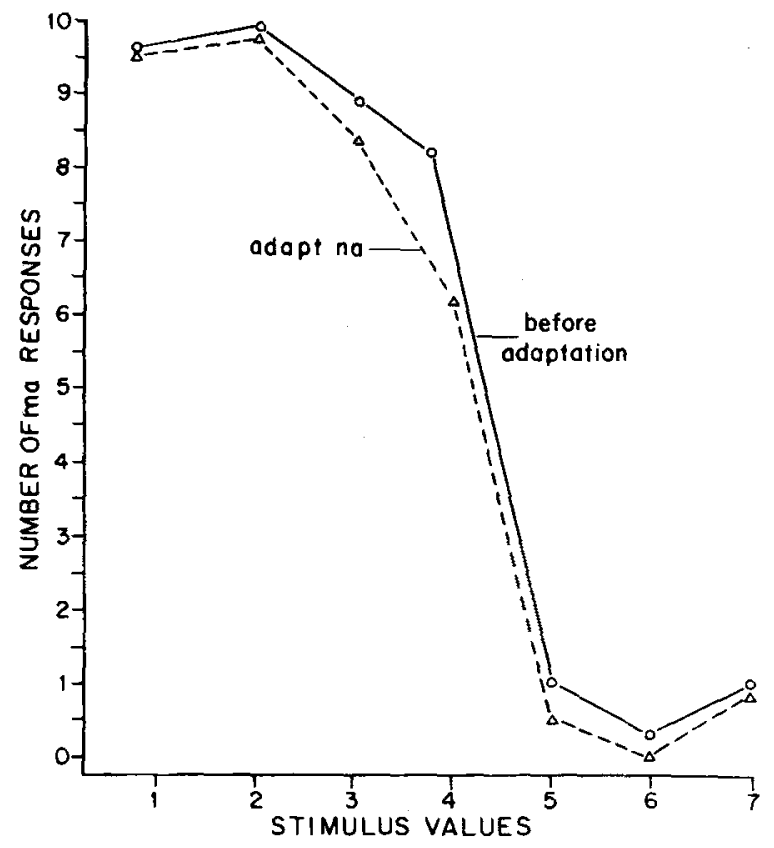

Figure 17. Mean number of [ma] responses to stimuli in the [ma]-[ba] series before and after repeated presentation of [na].

isolation. Previous experiments (e.g., Diehl, 1975; Eimas \& Corbit, 1973) have failed to produce adaptation effects using acoustic features presented in isolation. However, in these experiments, which employed synthetic speech, the acoustic feature was very short (e.g., $50 \mathrm{msec}$ ) and did not sound speechlike, since the original consonant was not a continuant, i.e., a segment that could be sustained in real speech. We decided to use a fairly long segment of nasal resonance $(150 \mathrm{msec})$, which was excised from a carefully articulated syllable. Although segments of nasal resonance prior to the vowel transitions in a CV syllable do not usually reach this duration in conversational speech, it should be emphasized that the original syllable did not sound unnatural and was clearly heard as [ma] before the nasal component was removed from before the vowel. The excised [m] noise when heard on the tape loop was clearly identifiable as a nasal-like speech sound. Experiment VII was performed to test for selective adaptation using this acoustic feature as the adapting stimulus.

\section{Method}

Subjects. Thirteen students enrolled in an introductory psychology course at Carnegie-Mellon University served as subjects. The subjects were tested in groups of three or four in a single 45-min session in a small sound-attenuated room.

Stimuli. The stimuli presented for identification were identical in Experiments V. VI, and VII. The order of presentation of syllables in the initial identical task and in the adaptation trials was also identical in the three experiments. In the present experiment, the adapting stimulus was a $150-\mathrm{msec}$ nasal noise removed from a 
naturally spoken [ma]. The presentation rate of the adapting noise was again three repetitions every 2 sec for $2 \mathrm{~min}$.

Procedure. The procedure was identical to the previous experiment.

\section{Results}

Figure 18 reveals a small but significant shift in the phoneme boundary of the [ma]-[ba] continuum following repeated presentation of the $[\mathrm{m}]$ noise ( $p<.01$ for $t$ tests based on area under the curve and $50 \%$ crossover point). It can be seen that the magnitude of the adaptation effect was almost identical for the [na] adapter in the previous experiment, and $[\mathrm{m}]$ noise adapter in the present experiment.

\section{EXPERIMENT VIII}

Previous experiments demonstrated selective adaptation with stop, affricate, and nasal consonants. Experiment VIII employes the same adaptation procedure with the voiceless fricative $[\mathrm{f}]$ in a [fa]-[ba] series.

\section{Method}

Subjects. Twelve students from the University of Waterloo served as subjects in two $1 / 2-h$ sessions 2 days apart. Subjects received $\$ 2$ for their participation.

Stimuli. The original [fa] used to construct the test series contained $150 \mathrm{msec}$ of frication and $232 \mathrm{msec}$ of transition plus vowel. So that all syllables in the series would have an abrupt onset, the first [fa] in the series, shown at the far left of Figure 19, had $13 \mathrm{msec}$ of frication spliced from the original [fa], leaving $137 \mathrm{msec}$ of frication prior to the vowel transitions. Each successive syllable in the series shown in Figure $19 \mathrm{had}$ an additional $22 \mathrm{msec}$ removed from its onset, so that Syllable 2 contained $115 \mathrm{msec}$ of frication and Syllable 7 on the far right of Figure 19 contained only $5 \mathrm{msec}$ of frication prior to the vowel transitions. Probably because of the abrupt onset introduced by tape splicing, subjects reported that some syllables (especially those near the boundary) sounded like [pfa]. However, subjects did not report any difficulty classifying each syllable as either [f] or [b]. The identification and adaptation trials using Stimulus 1 ([fa]) and Stimulus 7 ([ba]) as the adapting stimuli, were identical in all respects to the previous experiment.

Procedure. The procedure was also identical to that of previous experiments, except that subjects wrote either " $f$ " or " $b$ " to each test syllable. In the present experiment, and in all further experiments, subjects were presented all stimuli over loudspeakers.

\section{Results}

The results of Experiment VIII are shown in Figure 20. Unlike the previous experiments, adaptation with [fa], the first stimulus in the series, did not produce a shift in the phoneme boundary. Adaptation with [ba], however, produced a small, but significant, shift in the boundary in the predicted

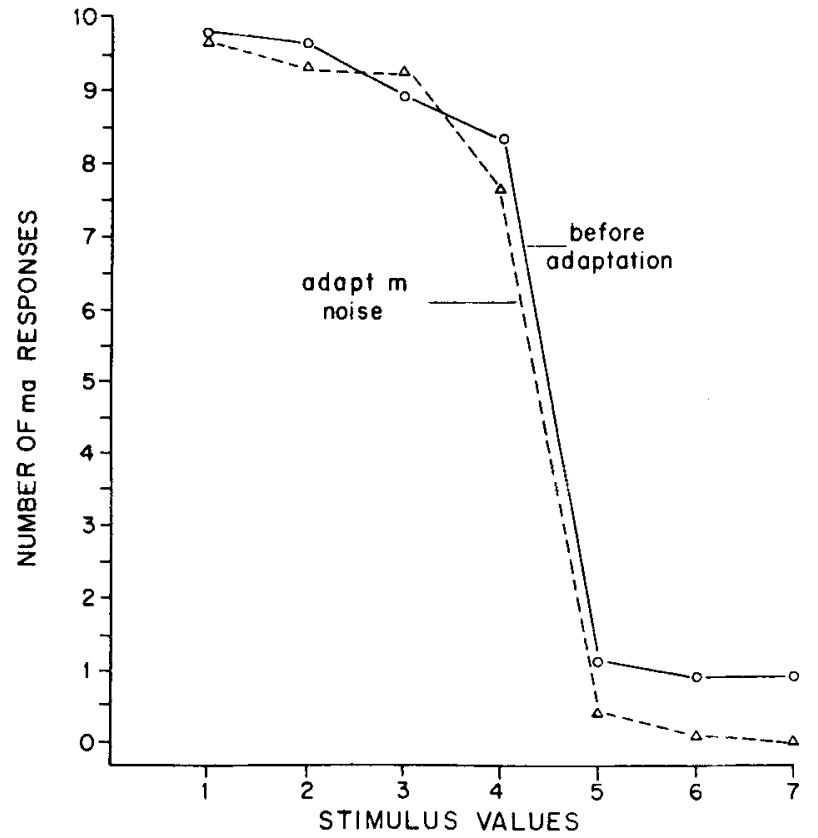

Figure 18. Mean number of [ma] responses to syllables along the [ma]-[ba] continuum before and after repeated presentation of 150 msec of nasal resonance excised from [ma].

direction $(\mathrm{p}<.05$; all further $t$ tests are based on area under the curve only).

\section{EXPERIMENT IX}

Repeated listening to [fa] did not result in a selective decrease in [fa] responses for syllables near the boundary on the [fa]-[ba] continuum. This result is surprising, since the results of the preceding experiments led us to believe that at least a small shift should have occurred based on adaptation at a phonetic level of analysis. It should be noted, however, that [fa] (along with [ $[\mathrm{a}]$ and [ha]) has one of the weakest energy spectra of any consonant phone. When [fa] was repeatedly presented to subjects, almost all subjects reported hearing the fricative noise as a soft "background hiss" after very few repetitions, and simultaneously heard the embedded syllable [ba] cued by the transition-vowel unit. The fact that subjects did not perceive the syllable [fa] most of the time during the periods of repetition may account for the lack of selective adaptation at the phonetic level.

Because of the failure to observe an adaptation effect for [fa], we decided to test for adaptation with [va]. While [fa] and [va] have a similar fricative

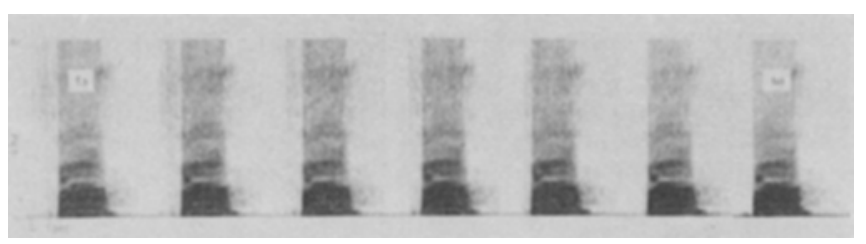

Figure 19. Speech spectrograms of stimuli in the $[\mathbf{f a}]-[\mathbf{b a}]$ series. 


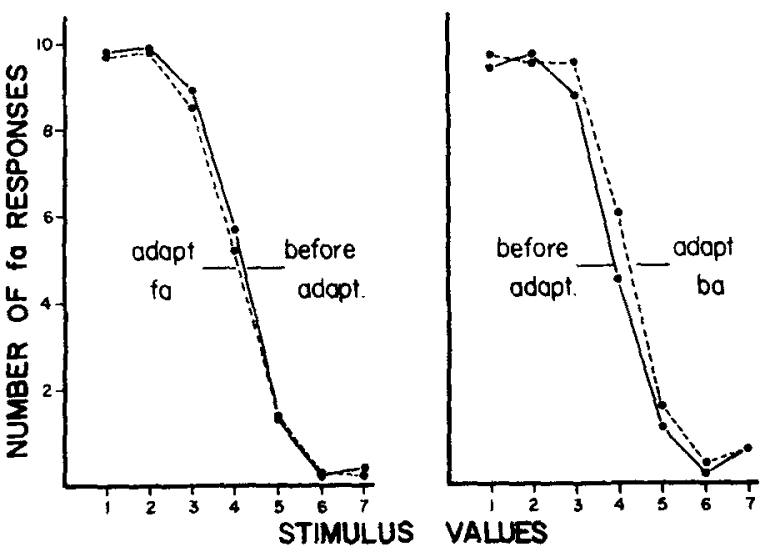

Figure 20. Mean number of [fa] responses to stimuli in the $[\mathrm{fa}]-[\mathrm{ba}]$ series before and after adaptation with [fa] and [ba].

component, [va] is accompanied by resonant energy in the lower frequencies. If the failure to obtain an adaptation effect for [fa] was due to the extremely low amplitude of the fricative portion of the syllable, we might expect an adaptation effect with [va], since the energy spectrum of [va], due to the voicing component, is considerably greater than for [fa].

\section{Method}

Subjects. Sixteen students from the University of Waterloo served as subjects in two $1 / 2-h$ sessions. Each subject was paid $\$ 2$ for his participation. Subjects were tested in groups of four in a small sound-attenuated room with the experimenter.

Stimuli. The original [va] contained $110 \mathrm{msec}$ of energy prior to the vowel transition. Since a normal [va] has a gradual onset time, the first [va] in the series had its initial $44 \mathrm{msec}$ removed, leaving $66 \mathrm{msec}$ of frication and voicing prior to the vowel transitions. Thus all syllables in the series contained an abrupt onset. The seven test syllables shown in Figure 21 were constructed by removing segments of $11 \mathrm{msec}$ from the successive syliables, so that Stimulus 7 on the far right of Figure 21 consisted entirely of a transition-vowel unit sufficient to cue [ba].

Seventy identification trials were followed immediately by seven adaptation trials on each day. As before, each adaptation trial consisted of 2 min (180 presentations) of either [va] or [ba] (depending upon the day) followed by 10 syllables for identification as $[v]$ or $[b]$.

Procedure. The procedure was identical to that of the previous experiments, except that subjects were instructed to write " $v$ " or " $\mathrm{b}$ " on their answer sheets when identifying the test syllables.

\section{Results}

The results, displayed in Figure 22, reveal the typical asymmetrical adaptation effect observed for the $[\mathrm{k}]-[\mathrm{p}],[\mathrm{j}]-[\mathrm{d}]$, and $[\mathrm{m}]-[\mathrm{b}]$ series. Repeated listening to [va] produced a selective decrease in [v] responses for syllables at the phoneme boundary $(\mathrm{p}<.01)$, while repeated listening to $[\mathrm{ba}]$ produced a smaller effect just short of significance $(p<.06)$. The magnitude of the shift was greater for [va] than for [ba] $(\mathrm{p}<.01)$.

\section{DISCUSSION}

These experiments demonstrate selective adaptation for stop ([b], [d], [p], [k]), nasal ([m]), affricate (ij]), and fricative ([v]) consonants. For acoustic continua spanning the phonemes $[\mathrm{k}]-[\mathrm{p}],[\mathrm{j}]-[\mathrm{d}]$, $[\mathrm{m}]-[\mathrm{b}],[\mathrm{f}]-[\mathrm{b}]$, and $[\mathrm{v}]-[\mathrm{b}]$, repeated presentation of the end-point syllable in each series resulted in a significant shift in the phoneme boundary in 8 of 10 eases (while $\mathrm{p}<.06$ for the [ba] adapter on the [va]-[ba] series). Only the [fa] adapter failed to produce evidence for selective adaptation along the [fa]-[ba] continuum.

When an effect is particularly robust, negative results tend to become most informative. It was with some delight, therefore, that we observed no significant decrease in [fa] responses along the [fa]-[ba] continuum following repeated presentation of [fa]. To our knowledge, this is the first failure to observe selective adaptation using an adapting syllable which was also present in the test series. All other failures to demonstrate selective adaptation (e.g., Ades, 1974b) have used adapting stimuli which differed in some way from the syllables in the test series.

It is comforting to note that selective adaptation cannot be observed for all syllables. Our negative result suggests, first, that adaptation is probably not occurring at the syllable level, since [fa] is psychologically as real as any of the syllables which produced an effect. Secondly, the failure to observe adaptation with [fa] strongly suggests that positive results in other experiments are not due to response bias. If adaptation were caused by some kind of response bias, a positive result should have been observed for [fa].

But why was there no effect for [fa], when a strong adaptation effect was observed for all of the other adapting stimuli? One plausible explanation is that there is no group of cells in the human auditory system which responds maximally to the consonant noise in [f]. This might occur because the frequency spectra are quite similar for [f] and [s], the main difference being that relative to the following vowel, [s] is more intense than [f] (Hughes \& Halle, 1956; Strevens, 1960).Perception of both [s] and [f] might therefore be mediated by a single group of cells which respond maximally to $[\mathrm{s}]$ and with reduced vigor to 


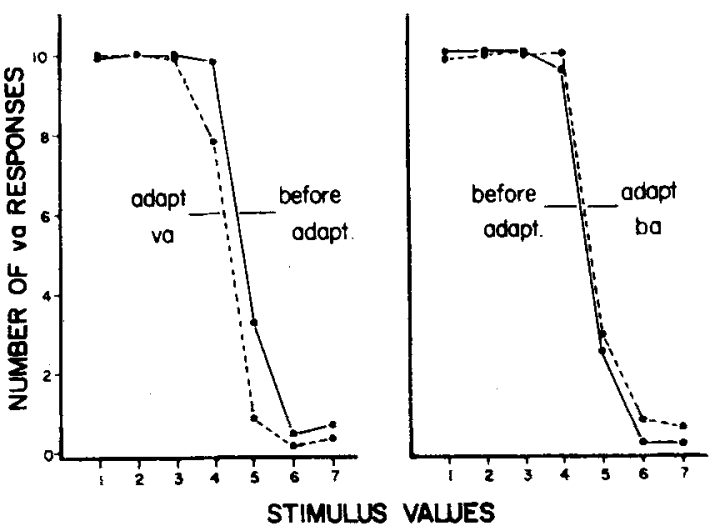

Figure 22. Mean number of [va] responses to stimuli in the [va]-[ba] series before and after repeated presentation of [va] and [ba].

[f]. If this hypothesis is correct, adaptation would occur along a [fa]-[ba] continuum following repeated presentation of [sa], but not [fa], since only [s] will maximally stimulate-and thereby fatigue-the feature detectors responsible for perception of [f]. This hypothesis is now being tested in our laboratory.

Our results suggest that adaptation is clearly selective, in that repeated presentation of a syllable always affected categorization of syllables lying near the phoneme boundary to a greater extent than syllables at the end points of the continuum. This result was also observed in the reaction time experiment. Repeated presentation of [ja] resulted in a shift in the peak of the reaction time function (which occurred at the phoneme boundary) but did not increase reaction times to the initial syllables in the continuum.

Several different results in the present experiments suggest that selective adaptation is determined mainly by the acoustic properties of the adapting and test stimuli. The fact that identical adaptation effects were observed along the [ja]-[da] continuum for [ja], [da], [ji], and [di] adapters suggests that the effect does not occur at a syllable level of analysis, but must occur at either a phonetic or acoustic level. Experiments with the [ma]-[ba] continuum suggest that the effect is partially phonetic and partially acoustic. Both the [na] and [m] noise adapter produced a reduced but significant shift in the phoneme boundary of the [ma]-[ba] series compared to the [ma] adapter. The significant effect for the [na] and [m] noise adapter demonstrates that adaptation does occur at the acoustic level; the reduced effect suggests a phonetic component.

A second major result of the present experiments was the asymmetrical adaptation effect. For all continua except [fa]-[ba], repeated presentation of the first syllable in the series produced a stronger adaptation effect than the final embedded syllable, although both adapting syllables produced a significant shift in the phoneme boundary in the predicted direction.

It is likely that asymmetrical adaptation is not merely due to the manner in which syllables in the test series were produced in our experiments. It could be argued, for example, that the first syllable in each series was somehow more "natural" than the final embedded syllable, since the embedded syllable was produced by tape splicing. It should be remembered, however, that all syllables were spliced into and therefore had an abrupt onset in the [ma]-[ba] and [va]-[ba] series, and a clear asymmetrical adaptation effect was found for both of these series. Further, one-sided adaptation was observed when [ii] and [di] were the adapting syllables, and both of these were unaltered, naturally spoken syllables. It seems unlikely, then, that asymmetrical adaptation reflects a difference in the naturalness of the two adapting stimuli.

We believe that asymmetrical adaptation provides additional evidence for a strong acoustic component to selective adaptation, and a weaker phonetic component. All of the test series in our experiments were constructed by removing increasingly longer segments of energy from a CV syllable. The acoustic change in each series from the original to the embedded syllable may therefore be viewed as the progressive loss of an acoustic feature (or features). For example, the change from [ma] to [ba] may be viewed as the progressive loss of the nasality feature, represented acoustically as nasal resonance in the lower frequencies.

We may view the process of categorizing syllables in the test series as a decision about the presence of a particular feature or set of features. For example, subjects identifying syllables in the [ma]-[ba] series must determine if the presented syllable has sufficient nasal resonance to be classified as [ma]. If classification of the test syllables depends upon perception of a particular acoustic feature, then adaptation should occur when the first syllable in each series is the adapting stimulus, since this syllable contains the perceptually important feature. Thus, repeated presentation of [ma] should produce a strong adaptation effect, since the subject repeatedly hears (and presumably becomes adapted to) the nasality feature. Following repeated listening to [ma], syllables in the test series which were previously heard as ambiguous should now be perceived as having less of the nasality feature, and should now be classified as [ba].

If adaptation occurred only at the acoustic level, there should be no shift in the phoneme boundary following repeated presentation of the embedded stop consonant, since the stop consonant lacks the acoustic feature which is responsible for identification of the 
other consonant phoneme in the series. The small but remarkably consistent adaptation effect observed in all experiments suggests a phonetic component to selective adaptation.

What do the present series of experiments allow us to conclude about feature detectors for speech sounds? Perhaps the strongest statement that can be made about selective adaptation based upon our results is that repeated listening to speech sounds produces consistent shifts in the identification of stimuli along a phonetic continuum. The direction of the shift in identification responses is readily explained by theoretical accounts which postulate that feature detectors for speech sounds are fatigued by repeated presentation of a particular sound (Cooper \& Nager, 1975; Eimas \& Corbit, 1973). However, these models have been postulated post hoc, and experiments have yet to be performed which test these models. For example, any model which attempts to explain selective adaptation using a "fatigue" model should also predict changes in sensitivity to stimuli following adaptation, and the appropriate signal detection experiments have yet to be performed.

An alternative and equally plausible explanation of selective adaptation is that repeated listening to a single speech sound narrows the range of stimuli which are now acceptable as belonging to the same phonetic category as the adapting syllable. ${ }^{6}$ Thus, a listener may normally accept a range from 70 to $30 \mathrm{msec}$ of frication as [ja] and 30 to $0 \mathrm{msec}$ as [da]. After listening to $2 \mathrm{~min}$ of the same [ja] having exactly $70 \mathrm{msec}$ of frication, the listener now restricts the acceptable range for [ja] to a lower limit of 40 (rather than $30 \mathrm{msec}$ ). According to this learning account of selective adaptation, the feature detector is "tuned" rather than fatigued by repeated listening to a single syllable.

The results of an experiment we recently performed suggest that the learning account of selective adaptation is inadequate. According to this account, adaptation should occur only when the adapting syllable provides the listener with a consistent example of a particular syllable, as in the present series of experiments. However, we observed a highly significant adaptation effect along a [ja]-[da] continuum when the repeated stimulus was actually a random repeating series of six syllables which varied from 60 to $25 \mathrm{msec}$ of frication. According to the learning account of adaptation, this variable adapting sequence should not have produced an adaptation effect, since the adapting sequence covered a wide range of frication.

It should also be noted that the learning account of selective adaptation cannot readily explain our asymmetrical adaptation results. If adaptation is caused by a tuning of the response mechanism after repeated listening to a single syllable, then the final embedded syllable in each of our test series should have provided an equally good definition of the target phoneme as the first syllable in each series, and therefore should have produced an equally strong adaptation effect. Asymmetrical adaptation is thus difficult to explain using the perceptual tuning hypothesis, but is more easily explained by a fatigue mechanism that operates mainly, but not exclusively, at an acoustic level of analysis.

\section{REFERENCE NOTES}

1. Carlson, R., Granstrom, B., \& Pauli, S. Perceptive evaluation of segmental cues. The 1972 Conference on Speech Communication and Processing, April 24-26, 1972; Boston.

2. Fischer-Jorgenson, E. Tape cutting experiments with Danish stop consonants in initial position. Annual Report VI. Copenhagen: University of Copenhagen, Institute of Phonetics, $1972,75-176$.

\section{REFERENCES}

AdEs, A. E. Bilateral component in speech perception? Journal of the Acoustical Society of America, 1974, 56, 610-616. (a)

AdEs, A. E. How phonetic is selective adaptation? Experiments on syllables position and vowel environment. Perception \& Psychophysics, 1974, 16, 61-66. (b)

Cole, R. A., \& Cooper, W. E. The perception of voicing in English affricates and fricatives. Joumal of the Acoustical Society of America, 1975, in press.

Cole, R. A., \& ScoTT, B. Toward a theory of speech perception. Psychological Review', 1974, 81, 348-374. (a)

Cole, R. A., \& ScotT, B. The phantom in the phoneme: Invarient cues for stop consonants. Perception \& Psychophysics, 1974, 15, 101-107. (b)

COOPER, W. E. Adaptation of phonetic feature analyzers for place of articulation. Journal of the Acoustical Society of America, 1974, 56, 617-627. (a)

CoOper, W. E. Contingent feature analysis in speech perception. Perception \& Psychophysics, 1974, 16, 201-204. (b)

CoOper, W. E., \& Blumstern, S. A "labial" feature analyzer in speech perception. Perception \& Psychophysics, 1974, 15, $591-600$.

Cooper, W. E, \& NAGER, R. M. Perceptuo-motor adaptation to speech: An analysis of bisyllabic utterances and a neural model. Journal of the Acoustical Society of America. 1975. 58, 256-265.

DienL, R. L. The effects of selective adaptation on the identification of speech sounds. Perception \& Psychophysics, 1975, 17, 48-52.

Eimas, P., \& Corbit, J. Selective adaptation of linguistic feature detectors. Cognitive Psychology, 1973, 4, 99-109.

Gerstman. L. Perceptual dimensions for the friction portion of certain speech sounds. Unpublished doctoral dissertation, New York University (Psychology), 1957.

Grimm, W. A. Perception of segments of English spoken consonant-vowel syllables. Journal of the Acoustical Sociery of America, 1960, 40, 1454-1461.

Hughes, G. W., \& Halle, M. Spectral properties of fricative consonants. Journal of the Acoustical Society of America, 1956, 28, 303-310.

Huntington, D., \& Miller, C. Certain speech/nonspeech perceptual contrasts. Journal of the Acoustical Society of America, 1972, 52, 112. (Abstract) 
MAlÉcot, A. Acoustic cues for nasal consonants. Language, 1956. 32. 274.284.

Pisoni, D. B., \& TAsH, J. Reaction times to comparisons within and across phonetic categories. Perception \& Psychophysics, $1974,15,285-290$.

ScotT, B. The verbal transformation effect as a function of embedded sounds. Unpublished Masters thesis, University of Waterloo, 1971.

Scotr, B. Speech perception: $A$ theory and application. Unpublished doctoral dissertation, University of Waterloo, 1974.

Strevens, P. Spectra of fricative noise in human speech. Language and Speech, 1960. 31, 32-49.

\section{NOTES}

1. It should be noted that this procedure does have some inherent difficulties. It is true that, in some cases, successively splicing into a $\mathrm{CV}$ syllable produces a perfectly natural continuum which varies in only one feature. For example, a naturally spoken [ja] and a naturally spoken [da] both have an abrupt onset following a closure interval, and differ mainly by the duration of the burst prior to the vowel transitions. By cutting into a naturally spoken [ja], it is possible to produce a continuum which varies in discrete steps from [ja] to [da], and we may speak of this continuum as varying along a single feature-burst duration. For other consonants, however, splicing successively greater segments from the onset of the original syllable results in a change of more than one feature. Thus, cutting into [fa] to produce a [fa]-[ba] series produces a change from a fricative to a stop, but also a change from a voiceless to a voiced consonant. A more serious problem is that by successively splicing into a sound, we may introduce a new feature, since we may change a sound with a gradual onset into a sound with an abrupt onset. In fact. it is possible to circumvent this problem by cutting increasingly larger segments from the middle of the original consonant (i.e., backward from the onset of the vowel transition) and then closing up the gap (Cole \& Cooper, 1975), or by starting the series with a syllable which has had some energy removed, thus introducing an abrupt onset.
2. Note that the difference in the magnitude of the effect for the two adapting stimuli is determined by adding the net change for each stimuius. This is done because opposite shifts are predicted with the different adapting stimuli. For instance, fewer [ka] responses are expected following repeated presentation of [ka], and so area under the [ka] identification curve should decrease. Conversely, the area under the curve should increase following repeated presentation of [pa]. Thus, if the area under the curve decreases by 15 following [ka] adaptation and inceases by 10 following [pa] adaptation, we add the changes $(-15$ and +10$)$. The resultant, -5 , indicates that there was a greater change in the predicted direction for [ka] adaptation than for [pa] adaptation. If the sum of the two numbers is positive, this would indicate that there was a greater shift in the predicted direction for the [pa] adaptation as compared to the [ka] adaptation.

3. One-tailed tests were used for simple adaptation effects, since a shift in the phoneme boundary is predicted in the direction of the adapting stimulus. No predictions were made, however. concerning differences in the magnitude of the effect for the two adapting stimuli, so that two-tailed tests were used.

4. It is not possible to adequately distinguish these two possibilities on the basis of the identification data presented here; however, these two possibilities can be distinguished using a task in which we assess subjects' ability to detect weak-intensity [ja] vs. [da] signals embedded in noise, both before and after adaptation. Adaptation of a unary detector should result in reduced sensitivity to both [jal and [da] with a [ja] adapter, whereas with a binary system sensitivity should be lowered only for the adapted phoneme.

5 . There were only 14 instances where reaction times were not recorded, and these occurred equally often on the preadaptation and postadaptation trials. On 8 occasions, subjects reported that they had pushed the wrong response button; in this case, reaction time was not recorded, but the identification response was noted. On the remaining 6 occasions, the timer did not trigger because of mechanical malfunction.

6. We thank Martin Taylor, Paul Kolers, and Mike Posner for a discussion of this view.

(Received for publication January 3.1975; revision received June $12,1975$. ) 